\title{
Eine ,praktische Lücke“ im Beweis - Zur methodologischen Kritik des Konsequenzialismus und des Prinzips der maximierenden Rationalität
}

\author{
Jens Kertscher • Philipp Richter
}

Online publiziert: 23. Juli 2020

(C) Der/die Autor(en) 2020

Zusammenfassung Der Beitrag entfaltet eine grundsätzliche Kritik an konsequenzialistischen Ethiken. Unsere Kritik zielt auf den Nachweis, dass konsequenzialistische Ansätze einer methodischen Anforderung bei der Begründung eines Moralprinzips nicht gerecht werden, weil sie einen Begriff des Guten voraussetzen, ohne auf seinen epistemischen Status zu reflektieren. Es gelingt ihnen daher nicht, einen Begriff des Guten zu entwickeln, der gleichermaßen sowohl die Erkenntnis einer logischen Notwendigkeit als auch einer praktischen Relevanz zum Ausdruck bringen kann. Aus methodischen Gründen muss daher unklar bleiben, warum das, was als „gut“ gesetzt und erkannt wird, auch realisiert werden muss.

Wir gehen in vier Schritten vor: In einem ersten Schritt greifen wir mit dem Ziel der Verschärfung des Problems mit dem so genannten deontologischen Paradox eine der größten Herausforderungen für deontologische Ethiken auf. Wir wollen aber zeigen, dass dieses Paradox überhaupt nur formuliert werden kann, wenn bereits von einem Prinzip des Guten ausgegangen wird, ohne es in ein Verhältnis zur praktischen Vernunft des Handelnden zu setzen. Dazu rekonstruieren wir in einem zweiten Schritt Mills Beweis für das Nützlichkeitsprinzip des Utilitarismus, weil sich daran das methodische Problem einer Erkenntnis des Guten besonders gut herausarbeiten lässt. Im dritten Schritt entwickeln wir unter Rückgriff auf methodologische Überlegungen Kants aus der Kritik der praktischen Vernunft ein Argument, das zeigen soll, dass in keiner moralphilosophischen Argumentation ein Begriff des Guten einfach vorausgesetzt werden kann, ohne logisch primär die durch das Prädikat „gut“ er-

\footnotetext{
J. Kertscher $(\triangle)$

Institut für Philosophie, Technische Universität Darmstadt, Karolinenplatz 5, 64289 Darmstadt, Deutschland

E-Mail: jens.kertscher@tu-darmstadt.de

P. Richter

Institut für Philosophie I, Ruhr-Universität Bochum, Universitätsstr. 150, 44780 Bochum,

Deutschland

E-Mail: philipp.richter@rub.de
} 
kannte praktische Notwendigkeit in ein Verhältnis zum Handelnden zu setzen. Das, was überhaupt als gut erkannt werden kann, ist daher durch reflexive Urteile über den Modus des praktischen Erkennens zu bestimmen. Mit diesem Ergebnis wird in einem vierten Schritt das deontologische Paradox aufgelöst.

Schlüsselwörter Deontologie $\cdot$ Konsequenzialismus · Paradox der Deontologie · Mills Beweis des Utilitarismus · praktische Vernunft · praktische Notwendigkeit

\title{
On a Practical Gap-A Methodological Critique of Consequentialism and the Principle of Maximizing Rationality
}

\begin{abstract}
The paper unfolds a fundamental critique of consequentialist ethics. Our criticism aims to demonstrate that consequentialist approaches do not meet a methodological requirement in establishing a moral principle because they presuppose a concept of good without reflecting on its epistemological status. Therefore, these approaches are unable to develop a concept of the good, which expresses both the understanding of its logical necessity and its practical relevance. Due to methodological reasons, in the perspective of an agent it must remain unclear why what is set and recognized as "good" also must be done.

The article takes four steps: In the first step, we address one of the greatest challenges for deontological ethics with the aim of exacerbating the problem of knowing what is good using the so-called deontological paradox. We want to show, however, that this paradox only could be phrased if a principle of good is already assumed (without putting it in relation to the practical reason of the agent). In order to do this, we reconstruct Mill's proof of the principle of utility in a second step. By considering Mill's proof, the methodological problem of knowing what is good can be worked out explicitly. In the third step, we develop an argument based on Kant's methodological considerations in the Critique of Practical Reason with the aim of showing that no approach in practical philosophy can simply presuppose a concept of good without putting its practical necessity, which is recognized by using the predicate "good", in relation to the agent. That which can be recognized as good can therefore be determined only by reflective judgments about the mode of practical knowing. With this result, the deontological paradox is resolved in a fourth step.
\end{abstract}

Keywords Deontology $\cdot$ consequentialism - paradox of deontology $\cdot$ Mill's proof of utilitarianism - practical reason $\cdot$ practical necessity 
To later generations, much of the moral philosophy of the twentieth century will look like a struggle to escape from utilitarianism. We seem to succeed in disproving one utilitarian doctrine, only to find ourselves caught in the grip of another. I believe that this is because a basic feature of the consequentialist outlook still pervades and distorts our thinking: the view that the business of morality is to bring something about.

- Christine Korsgaard, The reasons we can share

\section{Zur Problemstellung: „Praktische Notwendigkeit“" als Erkenntnis und Praxis}

In moralphilosophischen Begründungsdiskursen wird oft übersehen, dass nicht nur Prinzipien bestimmt und begründet, sondern diese Prinzipien auch in ein Verhältnis zur praktischen Vernunft eines rational Handelnden gesetzt werden müssen. Die Aufgabe besteht darin zu zeigen, warum Handlungen, die unter ein als objektiv gut erkanntes Prinzip fallen, auch realisiert werden müssen. Damit ist das Problem der praktischen Notwendigkeit angesprochen. Praktisch notwendige Sätze, d.h. insbesondere auch moralische Pflichten und Gesetze, treten Handelnden mit dem Anspruch auf Notwendigkeit entgegen: Es ist nicht möglich, dass die gebotene Handlung nicht erfolgt. Dass dieser Anspruch auf eine eigene begründungslogische Aufgabe verweist, wird klar, wenn man beachtet, dass praktische Gesetze sich an Wesen richten, die ein Vermögen der freien Präferenzwahl haben. Es muss daher aus der Perspektive eines praktisch vernünftig Handelnden begrifflich immer möglich sein, sich zu dem Satz, der ein praktisches Gesetz als Forderung ausdrückt, in ein Verhältnis zu setzen und ihm aus Freiheit auch zuwider zu handeln. Andernfalls wäre der Satz kein praktisches Gesetz, sondern ein Naturgesetz, und die fragliche Notwendigkeit folglich auch keine praktische, die das vernünftige und freie Handeln betrifft, sondern eine physikalische.

Es geht mit anderen Worten darum, wie ein praktisches Müssen kohärent gedacht werden kann. Ohne diesen Begründungsschritt bliebe der Begriff der praktischen Notwendigkeit inkohärent, denn es bliebe dann offen, wie die praktische Erkenntnis des Guten notwendigerweise praktisch wirksam wird. Es wäre dann beiläufig, ob die geforderte Handlung ausgeführt wird, oder nicht. Diese Begründungsaufgabe stellt sich für jeden moralphilosophischen Ansatz, der mit dem Anspruch auftritt, moralische Pflichten zu begründen.

Zur Verdeutlichung des Problems scheint es uns wichtig, zwei Bemerkungen voranzuschicken. Erstens, handelt es sich bei diesem Problem ausdrücklich nicht um das so genannte Motivationsproblem, d.h. um die Frage, was einen Handelnden faktisch-psychologisch motivieren kann, das als gut Erkannte auch tatsächlich zu realisieren. ${ }^{1}$ Ebenso wenig geht es um die Klärung der Frage, wie es denkbar ist, dass eine gedankliche Inferenz die Kraft zur Initiierung eines raumzeitlich iden-

\footnotetext{
${ }^{1}$ In den Kontext dieser Problematik gehören Diskussionen über den Zusammenhang von normativen und motivierenden Gründen oder auch um die Unterscheidung zwischen internen und externen Gründen. Vgl.
} 
tifizierbaren Geschehens als Tätigkeit erzeugen könnte. Vielmehr geht es um die Beantwortung der Frage, warum ich das, was ich als gut einsehe, auch auf diese oder jene Weise tun muss. Zur Klärung dieser Frage stellt sich der Akteur in den Raum der Gründe und versucht, sich selbst und andere davon zu überzeugen, inwiefern ihn seine Einsicht zugleich nötigt, auf bestimmte Art tätig zu werden.

Zweitens, fassen wir die Erkenntnis des Guten als eine praktische Erkenntnis auf. Eine praktische Erkenntnis ist nicht das Resultat einer theoretischen Reflexion über praktische Gegenstände, sondern die Erkenntnis, auf welche Weise zu handeln ist. Diese Frage stellt sich aus der Perspektive eines praktisch- vernünftigen Handelnden und kann auch als Frage nach den Möglichkeitsbedingungen vernünftigen Handelns in reflexiver Einstellung formuliert werden.

Methodisch gilt es, aus dem Begriff eines praktisch-vernünftig Handelnden zu begründen, warum die als gut erkannte Handlung erfolgen muss. Denn die Erkenntnis des Guten soll, wie bereits bemerkt, keine bloß theoretische Einsicht sein, sondern ihrem Begriff nach praktisch wirksam sein können. Die Frage lautet also, wie eine notwendige Verbindung des Begriffs des objektiv Guten mit dem Vermögen der freien Präferenzwahl rationaler Akteure gedacht werden kann. Diese Aufgabe ist Teil des Begründungsproblems und bedarf zu seiner Lösung einer eigenen methodischen Reflexion zum Status des Begriffs des Guten.

Einer der wenigen, der dieses Problem erkannt und zum Gegenstand einer methodischen Reflexion gemacht hat, war Immanuel Kant. ${ }^{2}$ Nach Kant ist „gut“ ausdrücklich ein praktischer Begriff: Kant bestimmt ,gut“ als formalen Gegenstand einer praktischen Erkenntnis. ${ }^{3}$ Seine Strategie, die die Strategie aller deontologischen Ansätze ist, besteht darin, ausgehend von diesem praktischen Modus der Erkenntnis die Bedingungen der Erkenntnis des Guten reflexiv zum Akt seiner Erkenntnis aufzuweisen und als ein allgemeingültiges Prinzip des Handelns auszudrücken. ${ }^{4}$ So soll klargemacht werden, dass der Begriff des Guten als ein wesentlich praktischer Begriff mit dem Begriff der praktischen Notwendigkeit verbunden sein muss. Denn nur das, was im praktischen Sinne als notwendig erkannt wird, kann als objektiv gut gelten und muss daher auch realisiert werden. Methodisch zeigt sich dann, dass der Begriff des Guten nicht einfach vorausgesetzt, sondern von diesem Prinzip her bestimmt wird.

Diese begründungslogische Anforderung und die damit verbundene methodische Einsicht zum Status des Begriffs des Guten werden, wie angedeutet, oftmals nicht hinreichend berücksichtigt. In unserem Beitrag wollen wir zeigen, dass insbeson-

aber Bambauer (2019, 348, 359f.), der herausstellt, dass Korsgaard praktische Erkenntnis als ein Motivationsproblem diskutiert.

$2 \mathrm{KpV}$, AA V, $58 \mathrm{f}$. Vgl. Kants Bemerkungen in der Vorrede zur Grundlegung zur Metaphysik der Sitten (GMS, AA IV, 389). Mit der Unterscheidung zwischen Naturgesetzen und Gesetzen der Freiheit eröffnet er die Problematik, unter welchen Bedingungen eine Erkenntnis von praktischer Notwendigkeit operieren können muss. Die Werke Kants werden in diesem Aufsatz nach der Akademie Ausgabe mit folgenden Siglen zitiert: KpV = Kritik der praktischen Vernunft (Kant 1961); GMS = Grundlegung zur Metaphysik der Sitten (Kant 1903).

3 GMS, AA IV, 412: „Der Wille ist ein Vermögen, nur dasjenige zu wählen, was die Vernunft unabhängig von der Neigung als praktisch notwendig, d. i. als gut, erkennt."Vgl. KpV, AA V, $57 \mathrm{f}$.

${ }^{4}$ Vgl. Stephen Engstroms Rekonstruktion des Willens bzw. guten Willens als Wissen vom Guten, Engstrom (1997, 21-29). Vgl. auch Engstrom (2009, VIIf., XI; 98-121; 199). 
dere konsequenzialistische Theorien dieser Anforderung nicht gerecht werden und daher ein grundlegendes methodisches Defizit aufweisen. Es gelingt ihnen nicht, einen Begriff des Guten zu entwickeln, der gleichermaßen die Erkenntnis einer logischen Notwendigkeit als auch einer praktischen Relevanz des als gut Erkannten zum Ausdruck bringen kann. Wie wir, insbesondere im Rückgang auf Mills „Beweis“ des Nützlichkeitsprinzips, zeigen wollen, liegt das daran, dass konsequenzialistische Ansätze einen Begriff des Guten als letzten Zweck voraussetzen, ohne ihn begrifflich mit der praktischen Vernunft des Handelnden verbinden zu können. Wir wollen dieses Grundproblem konsequenzialistischer Ansätze in Auseinandersetzung mit Samuel Scheffler und John Stuart Mill herausarbeiten und zeigen, weshalb konsequenzialistisch-utilitaristische Theorien am Problem der Erkenntnis des Guten notwendig scheitern müssen.

Auch hierzu sind zwei erläuternde Bemerkungen erforderlich: Erstens, ist eine moralphilosophische Konzeption, nach dem Verständnis, das wir in diesem Beitrag zugrunde legen, dann und nur dann konsequenzialistisch, wenn sie beansprucht, den Begriff einer richtigen Handlung einzig und allein über den Begriff des Guten im Sinne der herbeigeführten wünschenswerten Sachverhalte definieren zu können. ${ }^{5}$ Die Bestimmung des Konsequenzialismus als moralphilosophische Konzeption, die für die Bewertung von Handlungen den Akzent auf Folgen legt, ist also nach der von uns zugrunde gelegten Definition zu unspezifisch. ${ }^{6}$ Akzeptiert man diese Definition, stellt sich das angezeigte methodische Problem jedoch zwingend, da ein (wie auch immer) bestimmter Begriff des Guten als Ausgangspunkt für die Begründung moralischer Pflichten methodisch vorausgesetzt ist. Unser Argument richtet sich gegen alle konsequenzialistischen Theorien, die unter diese Definition fallen, und identifiziert ein methodisches Defizit genau dieses begründungstheoretischen Anspruchs.

Zweitens, wenn wir unser Argument an Mills Beweis entwickeln, dann deshalb, weil man daran das angezeigte methodische Problem besonders gut herausarbeiten kann. Selbstverständlich folgt daraus nicht, dass alle konsequenzialistischen Ansätze auf Mills Beweis verpflichtet wären oder einen vergleichbaren Beweis voraussetzen würden. Konsequenzialistische Ansätze nach Mill verzichten auf einen Beweis und berufen sich auf die unbestreitbare Geltung des Standards maximierender Rationalität. Nach diesem Standard ist man immer dazu verpflichtet, die beste mögliche Option zu realisieren. ${ }^{7}$ Eine Implikation unserer methodischen Kritik ist, dass die

\footnotetext{
5 Mit dieser Definition des Konsequenzialismus folgen wir Wiggins (2006, $149 \mathrm{f})$.

6 Der Gedanke, dass in moralisch relevanten Entscheidungssituationen, also im Konkreten, auch Erwägungen von Folgen eine Rolle spielen müssen, ist ohnehin trivial und wird auch von deontologischen Ansätzen nicht bestritten.

7 Das wird sehr gut deutlich in George Edward Moores konsequenzialistischer Definition von Pflicht: „Unsere ,Pflicht“ kann deshalb nur definiert werden als diejenige Handlung, die mehr Gutes in der Welt zustande bringen wird als jede mögliche Alternative. Und was ,richtig ' oder ,moralisch zulässig ' ist, hebt sich hiervon nur ab als das, was nicht weniger Gutes zustande bringen wird als jede mögliche Alternative. Wenn also die Ethik sich anheischig macht zu versichern, daß gewisse Handlungsweisen ,Pflichten“ sind, so macht sie sich anheischig zu versichern, daß solches Handeln immer die größtmögliche Summe des Guten hervorbringen wird."(Moore 1970, 211).
} 
Berufung auf diesen Standard nicht ausreicht, um das Problem der praktischen Notwendigkeit zu lösen.

Aus diesem Grund greifen wir in einem ersten Schritt mit dem Ziel der Verschärfung des Problems mit dem sog. deontologischen Paradox eine der größten Herausforderungen auf, die seitens konsequenzialistischer Theoretiker gegen deontologische Ansätze vorgebracht wurden. Wir wollen zeigen, dass dieses Paradox überhaupt nur formuliert werden kann, wenn von einem Prinzip des Guten ausgegangen wird, ohne es in ein Verhältnis zur praktischen Vernunft des Handelnden zu setzen. Dazu rekonstruieren wir in einem zweiten Schritt Mills „Beweis“ für das Nützlichkeitsprinzip des Utilitarismus. Vor dem Hintergrund des Ergebnisses unserer Auseinandersetzung mit Mill werden wir in einem dritten Schritt unter Rückgriff auf Kants methodologische Überlegungen aus der Kritik der praktischen Vernunft ein Argument entwickeln, das zeigen soll, warum jede konsequenzialistische Begründung eines Moralprinzips das von uns identifizierte methodische Defizit aufweist. Wenn diese Argumentation überzeugt, kann schließlich in einem letzten Schritt auch das deontologische Paradox abgewiesen werden.

\section{Praktische Notwendigkeit und das ,,deontologische Paradox“}

Den Hintergrund für unsere Diskussion bildet ein Angriff gegen deontologische Ethiken, der von Samuel Scheffler vorgebracht und unter dem Titel „Paradox der Deontologie“" bekannt geworden ist. ${ }^{8}$ Vor dem Hintergrund einer konsequenzialistischen Konzeption will Scheffler zeigen, dass deontologische Einschränkungen für Handlungen (agent centred restrictions) insofern paradox erscheinen, als Situationen denkbar sind, die es als geboten, also auch als vernünftig erscheinen lassen, die schlechtere Alternative der besseren vorzuziehen. Samuel Scheffler formuliert das Paradox folgendermaßen:

For how can it be rational to forbid the performance of a morally objectionable action that would have the effect of minimizing the total number of comparably objectionable actions that were performed and would have no other morally relevant consequences? How can the minimization of morally objectionable conduct be morally unacceptable ${ }^{9}$

\footnotetext{
8 Vgl. Scheffler (1991, insbesondere Kap. 4). Das von Scheffler aufgegriffene Problem geht zurück auf Nozick (2013, 30). In den an Schefflers Problemdiagnose anschließenden Diskussionen sind verschiedene, auf grundsätzlichen Überlegungen beruhende Versuche unternommen worden, das Paradox der Deontologie aufzulösen, bspw. mit Rückgriff auf das Prinzip der Doppelwirkung oder mit Rekurs auf Rechte von Personen. Weitere Vorschläge finden sich bei Kamm (2007), sowie Scanlon (2000). Schefflers Paradox fügt sich in eine Diskussion um moralische Dilemmata ein, die seit den 1970er Jahren intensiv geführt wird und nicht zuletzt auch auf die Frage hinausläuft, wie sich solche Dilemmata auflösen lassen, ohne den Konsequenzialismus schon voraussetzen zu müssen. Für einen neueren Beitrag vgl. Heuer (2011). Die Autorin versucht Akteur-zentrierte Einschränkungen ausgehend vom Versprechen zu rechtfertigen. Auf diese Diskussionen, die in erster Linie um konkrete Anwendungsfälle deontologischer Einschränkungen kreisen, gehen wir in unserem Beitrag nicht ein.
}

9 Scheffler 1985, 409; vgl. dort auch 413, 415. 
Scheffler legt großen Wert darauf, dieses Paradox so zu formulieren, dass nicht schon die Gültigkeit des Konsequenzialismus vorausgesetzt wird. Das einzige, was vorausgesetzt werden muss, ist ein allgemeiner, auch von Gegnern des Konsequenzialismus akzeptierter Rationalitätsstandard: Wenn ein rationaler Akteur ein Ziel als wünschenswert bewertet und vor zwei Optionen steht, von denen eine das Ziel herbeiführen kann, dann ist es für ihn ceteris paribus rational, die Handlung zu wählen, die geeignet ist, dieses Ziel herbeizuführen (Scheffler 1985, 414). Es ist mit anderen Worten immer rational, diejenige Handlung auszuführen, welche relativ zu den verfügbaren Optionen am besten geeignet ist, das als wünschenswert bewertete Ziel zu erreichen. ${ }^{10}$ Scheffler stellt dieses Rationalitätsmodell einer von Kants kategorischem Imperativ her gedachten Form praktischer Vernunft gegenüber, die dann als eine vom Standardmodell abweichende Form praktischer Rationalität erscheint und daher auch eigens begründet werden müsste. ${ }^{11}$

Aus deontologischer Sicht könnte man schon einwenden, dass jemand einen aus einem Prinzip hergeleiteten Grund haben kann, eine Tötung zu unterlassen. Daraus folgt nicht, dass er dann auch Gründe hat, das Vorkommen einer größeren Zahl von Tötungen zu verhindern. In diese Richtung argumentiert Thomas Scanlon. ${ }^{12} \mathrm{Im}$ Hinblick auf Schefflers Paradox folgt aus seinen Überlegungen, dass ein Handelnder, der das Tötungsverbot als Prinzip akzeptiert, dieses Prinzip bzw. die Gründe, die es generiert, nicht noch einmal abwägen muss gegen das Gute, das durch Verletzung dieses Prinzips realisiert würde. ${ }^{13}$

In unserem Beitrag wollen wir Schefflers Herausforderung zum Anlass nehmen, den Anspruch des konsequenzialistischen Modells auf Allgemeingültigkeit anzufechten. Wir wählen dabei allerdings einen anderen Weg als Scanlon. Wie schon

\footnotetext{
${ }^{10}$ Mit dieser Annahme ist eine Formulierung des Paradoxes möglich, die nicht auf die schlechten Folgen der zu meidenden Handlungen Bezug nimmt, sondern die Handlungen selbst als Bestandteile der zu bewertenden Sachverhalte einbezogen werden. Die Tatsache, dass in Schefflers Beispielen eine Handlung eine Tötung ist, erzeugt demnach einen schlechten Sachverhalt, so dass ein Akteur-neutraler Grund besteht, ihr Vorkommen zu meiden oder zu verhindern. Scheffler diskutiert mehrere Strategien, wie Verteidiger deontologischer Einschränkungen dem Paradox begegnen können, die seiner Auffassung nach aber alle scheitern. Diese Argumente müssen hier nicht ausführlich dargestellt werden. Vgl. Scheffler (1985, 414418).

${ }^{11}$ Scheffler $(1985,419)$ : „If these is a genuine Kantian view being challenged here, it is this: that it is possible to interpret the categorical imperative in such a way that it is plausibly thought of both as a requirement of practical reason and as supporting agent-centered restrictions in particular. This leaves much of what Kant thought about the relation of morality and rationality untouched. At the same time, the question it does raise is one to which the answer, I think, is not at all clear.“

${ }^{12}$ Scanlons Überlegungen unterlaufen Schefflers Herausforderung, indem er die Allgemeingültigkeit der konsequenzialistischen Identifikation von Werten mit einem allgemeinen Guten, das herbeizuführen ist, bestreitet. Diese Annahme trifft deshalb nicht zu, weil in vielen Entscheidungssituationen Handlungsgründe nicht ausschließlich auf der Erwägung von Folgen beruhen, sondern auf dem Vergleich von Gründen und ihrem Gewicht relativ auf die Situation. Scanlon macht hier auf die Komplexität von Entscheidungssituationen aufmerksam: Die Beachtung von Folgen ist nicht immer der ausschlaggebende Gesichtspunkt bei der Bewertung von Handlungsoptionen; die Bewertung einer Option als ,gut“ bedeutet nicht, dass der durch sie hervorgebrachte Sachverhalt sein soll; und: Aus der Tatsache, dass eine bestimmte Handlungsoption nicht gewählt wurde, folgt nicht, dass diese Handlung einen negativen Wert bekommt (Scanlon 2000, 80f.); zu Scheffler grundsätzlich (Scanlon 2000 82-83).

${ }^{13}$ Nach Scanlon ist für die Begründung dieses oder anderer Prinzipien der intrinsische Wert oder Unwert der Handlungen überhaupt irrelevant (Scanlon 2000, 85).
} 
angedeutet, liegt das Problem aus unserer Sicht weder in der teleologischen Konzeption praktischer Vernunft, noch in der formalen Bestimmung des Guten als das, was herbeigeführt werden soll. Diese Annahmen teilt auch Kant: Das teleologische Modell praktischer Vernunft und die Bestimmung des Guten als formalem Gegenstand der praktischen Vernunft. Unsere Überlegung ist daher die Folgende: Gerade weil das Gute das ist, was herbeigeführt werden soll, ist es nicht möglich, zu einer praktisch vernünftigen Antwort auf die Frage zu kommen, was zu tun ist, solange man methodisch vom Begriff des Guten ausgeht. Konsequenzialistische Theorien sind aber auf diesen methodischen Ausgangspunkt verpflichtet. Der Fehler liegt nach unserer Argumentation also weniger in der formalen Bestimmung des Guten als das, was hervorgebracht werden soll, sondern im falschen methodischen Ausgangspunkt. Als Antwort auf das Paradox der Deontologie nehmen wir eine Diskussion auf, die Kant als „Paradoxon der Methode in einer Kritik der praktischen Vernunft“ geführt hat (KpV, AA V, 62 ff.).

Das „deontologische Paradox“ resultiert, so lassen sich Schefflers Ausführungen verstehen, daraus, dass deontologische Argumentationen durch logische Ableitung aus einem Prinzip des Guten allgemeine Handlungstypen als gut oder schlecht klassifizieren, ohne diese Ableitungen wiederum ins Verhältnis zur praktischen Vernunft des Akteurs zu setzen. Jedoch sind bei Setzung eines Prinzips des Guten, so die Formulierung der Paradoxie, Situationen denkbar, die es absurder Weise als geboten und vernünftig erscheinen lassen müssten, die schlechtere Alternative - durch Beharren auf der ausschließlich erforderlichen Aktualisierung des guten Handlungstyps - einer eigentlich besseren Handlung vorzuziehen. Wenn dies zutrifft, dann würde es sich beim deontologischen Vorgehen in der Tat um eine seltsame Normierung der praktischen Vernunft durch die epistemische Autorität eines der praktischen Vernunft externen Prinzips handeln. Aufgrund der formulierten Paradoxie wäre dann jedoch die Frage berechtigt, warum gerade diese Setzung eines Prinzips und die entsprechenden Ableitungen daraus geeignet sein sollen, die Erkenntnis des Guten in praktisch vernünftiger Weise anleiten und sichern zu können.

\section{John Stuart Mills „Beweis“ für das Nützlichkeitsprinzip - Verteidigung und Kritik}

Scheffler setzt bei der Formulierung der Paradoxie den methodischen Ausgangspunkt vom Begriff des Guten voraus. Indem wir darauf aufmerksam machen, wollen wir zeigen, dass es sich bei der Frage nach einem Paradox der Deontologie gerade nicht, wie Scheffler annimmt, um ein rationalitätstheoretisches Problem handelt, wonach man dem Paradox nur entgehen könnte, wenn man eine vom Standard der maximierenden Rationalität abweichende Form praktischer Vernunft annimmt. Nach unserer methodischen Kritik am konsequenzialistischen Begründungsprogramm verschwindet das Paradox, wenn man den methodischen Ausgangspunkt konsequenzialistischer Ansätze beim Begriff des Guten aufgibt.

Das methodische Problem, das sich mit der konsequenzialistischen Begründung des richtigen Handelns aus einem vorausgesetzten Begriff des Guten ergibt, lässt sich paradigmatisch an John Stuart Mills Argumentation über die Methode der Be- 
stimmung des Guten als eines letzten Zwecks (ultimate end) darstellen. Ein Vernunftbeweis (proof by reasoning) dafür, ob eine Handlung gut oder schlecht sei, ließe sich, so Mill, nur führen, wenn von der Setzung eines Zwecks ausgegangen wird, von dem ohne Beweis zugegeben werde, dass er gut sei (Mill 2006, Kap. 4, 105; Kap. 1, 17.):

Whatever can be proved to be good, must be so by being shown to be a means to something admitted to be good without proof. (Mill 2006, 16)

Aufgrund der für einen Vernunftbeweis erforderlichen Relativierung der Ableitung auf die Setzung einer Prämisse, ließen sich daher weder letzte Zwecke (ultimate ends) noch erste Prinzipien (first principles) als gut bzw. wahr im Sinne eines deduktiven Beweises rechtfertigen (Mill 2006, 105); denn würden sie deduktiv bewiesen, wäre ihr Status als letzter Zweck bzw. erstes Prinzip damit zugleich widerlegt. Vernunftbeweise bleiben nach Mill also in praktischer Hinsicht restringiert auf Argumentationen für geeignete Mittel, die relativ gut für gesetzte Zwecke sind. Für den Nachweis, dass das Glück (happiness) ein letzter Zweck sei, ist daher eine andere Weise des Argumentierens erforderlich. ${ }^{14}$

Wenn wir uns nun im Folgenden dem Mill'schen Beweis des Nützlichkeitsprinzips zuwenden, wollen wir dabei weniger die Gültigkeit des Arguments immanent kritisieren, sondern vielmehr den Blick auf dessen Methode richten. Insbesondere werden wir nach dem Verhältnis fragen, in dem sich die praktische Vernunft des Akteurs und die Konklusion des Arguments, die bekanntlich den Nachweis des Glücks als eines letzten Zwecks erbringen soll, befinden. Schefflers Formulierung des deontologischen Paradoxes moniert den Prinzipiendogmatismus des deontologischen Vorgehens. Aber kann Mills Argumentation diesen Dogmatismus vermeiden?

Wir wollen im Folgenden Mills Beweis zunächst stark machen, indem wir ihn als Enthymem interpretieren. Wir grenzen uns dabei von üblichen Interpretationen $\mathrm{ab}$, die versuchen, Mills Beweis auf dem Boden seiner empiristischen Methodolo-

\footnotetext{
14 Mill stellt sich dabei in die Tradition der empiristischen Moralphilosophie. Bereits in David Humes Enquiry Concerning the Principles of Morals findet sich eine fast wortgleiche Formulierung hinsichtlich der Möglichkeit praktischer Beweise (Hume 1751/2003, 132f.). Der Unterschied besteht jedoch darin, dass nach Hume die der Ableitung des geeigneten Mittels vorausgehende Setzung eines Zwecks dem Akteur naturkausal determinierend, im Sinne eines Affekts, zustößt. Mill scheint dagegen, insbesondere wenn man seine Auffassungen in der politischen Philosophie mitberücksichtigt, ein zumindest psychologisch freies Disponieren über relativ gute Zwecksetzungen auf Grundlage intersubjektiver Verbindlichkeit anzunehmen (Wolf 2012, 139-141, 165-167, 217-225). Vgl. auch Brink (1992, 67-103, 78 ff.). Diesen Interpretationsansätzen zufolge sind die argumentativen Schwächen in Mills Beweis und die Unklarheiten hinsichtlich einer theoretischen und praktischen Bestimmung von Glück eigentlich als epistemische Tugend aufzufassen; nämlich als Ausdruck einer nicht fundamentistisch deduktiven, sondern kohärentistischen Argumentationstheorie der moralischen Urteilsbildung. Im Rahmen der liberalistisch-humanistischen Weltanschauung Mills würde dann in freier, gemeinsamer Auseinandersetzung danach gesucht, was menschliches Glück der Individuen oder der Gemeinschaft steigern würde (Heydt 2006, 58 ff., 153f.). Diesen Interpretationsansätzen kann jedoch nicht gefolgt werden, da sie Mills Beweis schwächer machen, als er eigentlich rekonstruierbar ist, und zudem in einen normativen Relativismus der Ableitung des richtigen Handelns führen. Wie wir zeigen wollen, bietet auch diese vermeintlich politisch liberale Interpretation des Mill'schen Beweises keinen Ausweg aus dem methodischen Grundproblem, das Mills Umgang mit dem Begriff des Guten zugrunde liegt und den Beweis notwendig scheitern lassen muss.
} 
gie zu rekonstruieren. ${ }^{15} \mathrm{Im}$ Gegensatz zu diesen Interpretationsansätzen soll Mills Argumentation im Folgenden als Präsuppositionsanalyse des Handlungsbegriffs rekonstruiert werden. Für diese unorthodoxe Herangehensweise sprechen aus unserer Sicht drei Gründe: Erstens, die gängigen empiristischen Deutungen lassen den Beweis schwächer erscheinen als nötig. Zwar gelingt es ihnen, Mill gegen den Vorwurf grober logischer Fehler zu verteidigen, allerdings müssen sie letztlich eingestehen, dass Mills „Beweis“ höchstens als eine Plausibilisierung verstanden werden kann. ${ }^{16}$ Dagegen soll unsere Rekonstruktion zeigen, dass der Beweis ein gültiges Argument darstellt. Aus der Konklusion, dass das Glück ein notwendiges Gut ist, lässt sich dann auch folgerichtig die Formel des Nützlichkeitsprinzips deduzieren. Mit diesem Rekonstruktionsvorschlag lässt sich, zweitens, die einseitige, philosophiegeschichtliche Klassifikation, entweder Empirismus oder Apriorismus, vermeiden. ${ }^{17}$ Drittens, nehmen wir mit der Präsuppositionsanalyse des Handlungsbegriffs den Ausgangspunkt bei der praktischen Perspektive eines rational handelnden Akteurs, der sich selbst oder andere von der Wahrheit des Nützlichkeitsprinzips überzeugen will. Anders als in den gängigen Deutungen erscheint der Beweis dann nicht als theoretisches Argument, sondern als Argument im Rahmen einer reflexiven praktischen Begründung. ${ }^{18}$

Wir kommen nun zur Rekonstruktion des Mill'schen „Beweises“. Das Argument stellt sich im Text des 4 . Kapitels von Utilitarianism wie folgt dar:
1. Prämisse
„Each person [...] desires his own happiness.“
2. Prämisse
Konklusion
„Each person's happiness is a good to that person."
„And the general happiness [is], therefore, a good to the ag- gregate of all persons." 19

Mill geht davon aus, dass ,,jeder sein eigenes Glück erstrebt“ (1. Prämisse) (Mill 2006, 107). Die Verwendung des Allquantors ,,jeder“ zeigt an, dass diese Aussage nicht empirisch-induktiv durch eine Verallgemeinerung von Selbst- und Fremdbeobachtungen begründet sein kann. Weiter gilt, nach Mills eigener methodischer Ausführung, dass es sich bei dieser Prämisse auch um keine bloße Setzung handeln darf, denn sonst würde die Konklusion per Vernunftbeweis deduktiv aus dieser Set-

\footnotetext{
${ }^{15}$ So ist vorgeschlagen worden, den Beweis als „schwache Letztbegründung“ im Sinne eines Hinweises auf eine psychologische Notwendigkeit zu deuten (Wolf 2012, 133, 157); andere wollen den Beweis auf das nicht nur rationale Fundament einer empirischen Evidenz gegründet sehen: Müller (2003, 176-182, 188) und Sayre-McCord (2001, 2-4). Außerdem Skorupski (1991, 285-299).

${ }^{16}$ So lassen sich die unterschiedlich akzentuierten Deutungen von Müller (2003), Sayre-McCord (2001) und Skorupski (1991) im Ergebnis zusammenfassen.

17 Vgl. Müller (2003, 187 ff.). Transzendentalphilosophische Ansätze fallen beispielsweise aus diesem Raster heraus.

${ }^{18}$ Für unterschiedliche Varianten, Mills Beweis als deduktives Argument darzustellen vgl. Fyfe (2012). Fyfe verteidigt dabei Mill gegen einige der gängigen Vorwürfe (naturalistischer Fehlschluss, Trugschluss der Komposition, äquivoke Verwendung von „desirable“), kommt allerdings zu dem Ergebnis, dass es auf der Basis der empiristischen Methodologie Mills keine in jeder Hinsicht zufriedenstellende Interpretationsmöglichkeit für den Beweis gibt, insbesondere habe Mill den Konsequenzialismus dogmatisch vorausgesetzt (Fyfe 2012, 226f.).

19 Vgl. Mill (2006, 106). Diese Darstellung des Arguments wird seit Sidgwick $(1981,388)$ allgemein akzeptiert.
} 
zung gefolgert und das Glück wäre daher kein letzter Zweck. Schließlich müsste bei einer deduktiven Ableitung im Voraus zugegeben worden sein, dass das Erstreben je meines Glücks notwendig gut ist. Wie können wir uns dann aber davon überzeugen, dass die 1. Prämisse wahr ist, ohne eine induktiv-empirische oder analytische Begründung, wobei letztere zudem wiederum die Annahme einer übergeordneten Prämisse erfordern würde, in Anschlag zu bringen? Inwiefern also ist die These, jede Person erstrebt ihr eigenes Glück, zutreffend?

Dem principle of charity folgend, schlagen wir vor, Mills Argument als ein Enthymem aufzufassen. Um die Begründung der 1. Prämisse und den Übergang zur 2. Prämisse rekonstruieren zu können, müssen unausgesprochene Annahmen des Arguments expliziert werden. Zunächst fällt auf, dass in allen drei Sätzen des Arguments über Präferenzurteile von Personen gesprochen wird. Unter welchen Bedingungen kann davon die Rede sein, dass eine Person mit Blick auf ihr Handeln wertende Urteile fällen kann, also z. B. ihr eigenes Glück als ,gut“ beurteilt? Um Mills Argumentation konsistent und kohärent auffassen zu können, ist an dieser Stelle eine Analogisierung mit dem argumentativen Vorgehen von Alan Gewirth in Reason and Morality hilfreich (Gewirth 1981).

Gewirth nimmt eine Präsuppositionsanalyse des Handlungsbegriffs aus der Innenansicht eines rational handelnden Akteurs vor, der sich in einer Reflexion gewissermaßen mit den Bedingungen der Denkbarkeit seiner Handlungsfähigkeit konfrontiert. ${ }^{20}$ Angenommen, ich verfüge über Handlungsfähigkeit, dann besteht nach Gewirth die erste Einsicht darin, die Notwendigkeit der Zweck-Mittel-Struktur des Handelns zu erfassen (Gewirth 1981, 48 ff.). Wenn wir nun weiter davon ausgehen müssen, dass jede Handlung einen Zweck hat, ergibt sich das Problem, dass sich jeder Zweck wiederum als Mittel für einen anderen Zweck denken lässt. Um zur Erläuterung der Problematik eine Formulierung des Aristoteles zu bemühen, wäre dann aber das Handeln als ein rationales, zielgerichtetes Streben ohne die Angabe eines letzten Zwecks, der nicht wiederum Mittel zu einem Zweck ist, ,sinnlos und leer“"21. Die gedanklich iterierende Struktur der relativen Mittel und Zwecke würde es nicht erlauben, ein erreichbares Ziel zu denken und die reflektierte Tätigkeit könnte nicht als Handeln, sondern nur als ungerichtetes Geschehen aufgefasst werden. Wenn Handeln jedoch nur als Tätigkeit mit (realisierbarem) Zweck identifiziert werden kann, dann muss zur Vermeidung des infiniten Regresses ein letzter Zweck angenommen werden. Diesen letzten Zweck (ultimate end) kann man mit Mill, was er in der 1. Prämisse auf den Begriff bringt, in der subjektiv-hedonistischen Perspektive eines endlichen Wesens als „Glück“ bezeichnen. Gewirth argumentiert ähnlich, jedoch ohne den Begriff eines ultimate end $\mathrm{zu}$ gebrauchen. Vielmehr, so Gewirth, kann der Handelnde einsehen, dass jeder singuläre, affirmierte Zweck zugleich eine Affirmation der Bedingungen der Handlungsfähigkeit einschließen

\footnotetext{
20 Gewirth bezeichnet die Perspektive dieser Reflexion als ,dialektisch“, um sie von einer gleichsam teilnahmslosen, objektbezogenen Reflexion von außen zu unterscheiden (Gewirth 1981, 42-47). Jean-Claude Wolf verweist in seinem Kommentar zu Mills Utilitarismus ebenfalls auf Alan Gewirth' Argumentation und die Analogisierungsmöglichkeit mit Mills Vorgehen hinsichtlich der Beweisführung für ein Moralprinzip in Akteursperspektive, verfolgt diese Interpretation jedoch nicht weiter (Wolf 2012, 24f.).

21 EN I, 1094a 21. Aristoteles' Nikomachische Ethik in der Übersetzung von Ursula Wolf zitiert als EN (Aristoteles 2006).
} 
muss (als ein generic good), sonst wäre das Erreichen dieses affirmierten Zweckes durch Aktualisierung der Handlungsfähigkeit nicht widerspruchsfrei denkbar (Gewirth 1981, 52-58). Nach Gewirth sind ,freedom“ und ,,wellbeing“ necessary goods für jeden Handelnden, insofern er praktisch vernünftig handelt, d.h. durch Mittel rational nach Zwecken strebt. ${ }^{22}$ Doch inwiefern genau ist das Streben nach Glück eine Präsupposition jedes Handelns? Warum ist nach Mill jede Person dazu genötigt, zuzugeben, dass sie nach Glück strebt?

Die Antwort findet sich in der 2. Prämisse. Mills Argument geht nach Formulierung der Prämisse 1 dazu über, den Akteur, der sich als nach seinem eigenen Glück strebendes Wesen versteht, darauf hinzuweisen, dass dieses Streben nach dem eigenen Glück für den Handelnden keine kontingente Zwecksetzung sei, sondern für diesen vielmehr ein Gut darstelle:

(2): Each person's happiness is a good to that person. (Mill 2006, 106)

Mit der substantivischen Formulierung ,,a good“ - im Unterschied z. B. zu dem Satz „happiness is good to that person“ - zeigt Mill in der 2. Prämisse die Einnahme einer abstrakt-objektivierenden Perspektive an, die der Handelnden auf seine Tätigkeit einnimmt. Glück ist für mich als Handelnden nicht nur ein kontingentes, sondern vielmehr ein notwendiges Gut. Wir wollen Mills Begründung der 2. Prämisse in Analogie zu Gewirth' Präsuppositionsanalyse des Handlungsbegriffs unter Abstraktion von den individuell-psychischen Merkmalen des Handelnden auffassen: Angenommen, ich bin ein handelndes Wesen, dann sind viele meiner Zwecke kontingent, nicht aber die ermöglichenden Bedingungen für Zwecksetzung und deren Erreichung - z. B. mein Wohlergehen bzw. my own happiness. ${ }^{23}$ Mills Konklusion, deren Folgerung im Text durch das logische Partikel ,therefore“24 anzeigt wird, stellt dann das Ergebnis einer semantischen und deduktiv gültigen Umformung der 2. Prämisse dar: Für jeden Einzelnen und somit für alle, insofern sie rational handlungsfähig sind, ist Glück ein Gut:

(K): the general happiness [is] a good to the aggregate of all persons. (Mill 2006, 106)

Damit wurde gezeigt, was zu „,beweisen“ war. Glück ist ein letzter Zweck, weil es für jeden Einzelnen bzw. für alle notwendig ein Gut ist. Diese Konklusion muss als eine Existenzbehauptung verstanden werden, die durch die vorherige Argumentation gesichert ist: Es gibt einen letzten Zweck und dieser ist das Glück. ${ }^{25}$ Der

\footnotetext{
22 Steigleder $(1997,255)$.

23 Nach Gewirth' Analyse der Struktur des Handelns ist es in einem ersten Schritt einsichtig, dass ,,a particular purpose“ vom Handelnden als ,,a good“ angesehen werden muss. In einem zweiten Schritt wird einsichtig, dass folglich im Allgemeinen auch Handlungsfähigkeit als ein good gelten muss - Gewirth markiert den zweiten bzw. Abstraktionsschritt mit dem Begriff ,a generic good“ (Gewirth 1981, 52).

24 Dieser dritte Satz im rekonstruierten Argument ist ersichtlicher Weise eine logische Schlussfolgerung bzw. eine Konklusion. Das ist im englischen Text eindeutig: ,,... and the general happiness, therefore, a good to the aggregate of all persons“ (Mill 2006, 106). Birnbacher übersetzt zutreffend mit „daher“ und weist dadurch den Satz auch im deutschen Text eindeutig als Schlussfolgerung aus. Es ist daher abwegig, wie Olaf L. Müller es tut, den zitierten Satz bzw. die Konklusion des Arguments als eine durch Mill hinzugefügte „moralisch wertende Behauptung“ zu bezeichnen (Müller 2003, 183).

25 Mills Rede von „,general happiness“ ist freilich mehrdeutig; sie lässt sich zum einen verstehen als ,,das Glück der Allgemeinheit“ (viele oder alle Menschen sind glücklich) oder zum anderen als das „Glück in unpersönlich allgemeiner Betrachtung“ („Glück“ im Sinne eines Allgemeinbegriffs für einen Zustand, der
} 
Nachweis dieses letzten Zwecks, als eines nicht nur subjektiv notwendigen, sondern objektiv notwendigen Gutes, erlaubt dann die Ableitung der Formulierung des Nützlichkeitsprinzips durch analytischen Vernunftbeweis (proof by reasoning). Es wäre ein logischer Widerspruch, Handlungen, die dem Nützlichkeitsprinzip zuwiderlaufen, als gut zu bezeichnen, da dieses angibt, was das beste Mittel zur Realisierung von Glück - im Sinne von dessen Maximierung in möglichst vielen Kategorien, z. B. Quantität, Qualität, Zeit, Ort - als einem notwendigen Gut darstellt. Alle anderen vermeintlichen letzten Zwecke bzw. Prinzipien des Guten, z. B. auch das der Deontologie, müssten als relativ geeignete Mittel zur Realisierung des Glücks aufgefasst werden. Genau darauf soll uns das von Scheffler formulierte deontologische Paradox hinweisen: Es müssten die als gut klassifizierten, allgemeinen Handlungstypen mit Blick auf die Optimierbarkeit ihrer Realisierung als Mittel zum letzten Zweck „Glück“ überprüft werden, damit sie als allgemeinverbindlich gut im Sinne der praktischen Vernunft des Akteurs erkannt werden könnten.

In methodologischer Absicht stellen sich nun die folgenden Fragen an unsere Rekonstruktion des „Beweises“: In welchem Verhältnis steht der praktisch vernünftige Akteur zur Erkenntnis, Glück ist als letzter Zweck objektiv notwendig ein Gut? Warum soll, so muss sich der Akteur fragen, die Realisierung des Glücks gut sein? Warum ist es praktisch vernünftig, nicht nur mein Glück, sondern das (unpersönliche) Glück zu realisieren? Vor dem Hintergrund der bisherigen Ergebnisse unserer präsuppositionsanalytischen Rekonstruktion sind zwei Antwortstrategien denkbar: (1.) Glück zu realisieren ist gut, weil Glück für den Handelnden subjektiv notwendig ein Gut ist - also etwas, das er in der Perspektive seines Wollens als gut beurteilen muss; oder (2.) Glück zu realisieren ist gut, weil Glück objektiv notwendig ein Gut ist - also etwas, das sein soll. Trifft nun Antwort (1.) zu, dann bleibt fraglich, warum es praktisch vernünftig sein soll, das Glück und nicht nur mein Glück zu realisieren. Letzteres widerspräche aber dem utilitaristischen Nützlichkeitsprinzip, das die Maximierung abstrakter Glückseinheiten in der Welt fordert. Das Problem der Antwortstrategie 1 besteht darin, dass aufgrund der Setzung der Beweis-Prämissen 1 und 2 ,mein Glück“ vom jeweils Handelnden als „,notwendig gut" beurteilt werden muss. Das ist auch nicht unplausibel, wie wir durch Stärkung des Beweises gezeigt haben. Allerdings muss demnach dann auch jede Präferenzwahl des Handelnden, die er in einem Urteil der Form ,, $\mathrm{x}$ ist gut (und besser als non-x)“ ausdrückt, letztlich durch Rückführung auf die Realisierung von ,,meinem Glück“ (bzw. je seinem/ihrem Glück) begründet werden. Dann wird jedoch der vermeintlich letzte Zweck ,das Glück“ im Handeln immer durch ein Präferenzurteil, dieses zu realisieren, subjektiv, überschrieben' und somit letztlich als ein nur relativ gutes Mittel zur Realisierung „meines Glücks“ begründet.

Trifft dagegen (2.) zu, dann bleibt fraglich, inwiefern mich der Nachweis der Existenz eines objektiv notwendigen Guts zu irgendeinem Handeln verpflichten sollte. Mills deduktive Ableitung der Formel des Nützlichkeitsprinzips aus der Konklusion seines Arguments müsste hier die Prämisse „Die Erkenntnis des objektiv notwendig Guten verpflichtet zu seiner Realisierung“ einführen. Wie jedoch wäre diese zu be-

qua Nützlichkeitsprinzip unparteiisch, unpersönlich oder altruistisch herbeigeführt werden soll). Letzteres ist die Bedeutung in der Konklusion. 
gründen? Ein proof by reasoning scheidet aus, da durch diesen der Status von Glück als eines letzten Zwecks aufgehoben würde. Man müsste nämlich bspw. argumentieren, dass Glück ein notwendiges Gut ist und alles notwendig Gute realisiert werden soll, weil das vernünftig ist bzw. ansonsten zu einem logischen Widerspruch führen würde. ${ }^{26}$ Dann aber wäre Glück als Mittel zur Realisierung von Vernunft relativiert und als Mittel ließe es sich ggf. gegen Alternativmittel verrechnen, die womöglich in höherem Maße zur Realisierung von Vernunft führen würden. „Glück“ muss Mills Argumentation zufolge jedoch als ein notwendiges Gut behandelt werden, das daher unvermittelt realisiert werden soll.

Eine andere, gleichsam dritte Antwortstrategie könnte die Betrachtung von Mills Ausführungen über die Methode des Existenznachweises von etwas Sichtbarem oder Hörbarem nahelegen:

The only proof capable of being given that an object is visible, is that people actually see it. The only proof that a sound is audible, is that people hear it. (Mill 2006, 104)

In Analogie hierzu könnte Mill auf die Unausweichlichkeit eines Strebens nach Glück hinweisen: „Du siehst, was Du auch tust, Du strebst nach Glück.“ Diese Strategie scheitert jedoch daran, dass das Vorliegen eines Strebens in normativer Hinsicht weder dessen Legitimität (als ein gutes Streben) noch die Legitimität bestimmter Weisen, nach Glück zu streben, als praktisch vernünftig rechtfertigen könnte. ${ }^{27}$

Haben wir hier nach beiden Antwortstrategien nicht ein Analogon zum sog. deontologischen Paradox erreicht? Ich soll etwas tun, ohne dass ich mich selbst von der praktischen Vernünftigkeit dieses Sollens, als etwas Gutem, überzeugen könnte? Mill hat keine Antwort, wie der Akteur mit den aufgrund des Handelns aus Freiheit notwendig auftretenden Warum-Fragen weiter umgehen soll. Vielmehr kann die oben rekonstruierte Argumentation nicht zeigen, warum es für den Akteur praktisch vernünftig sein muss, von der Einsicht in die Existenz des Glücks als eines notwendigen Guts zu dessen handelnder Realisierung im Sinne des Nützlichkeitsprinzips überzugehen.

Wir können nun das methodische Problem bzw. die ,praktische Lücke“ in Mills Beweis explizit benennen. Angesichts der Argumentation des Beweises muss sich ein Handelnder, wenn er die Prämissen 1 und 2 und die erforderliche Zusatzprämisse

\footnotetext{
26 Genauso sehen es auch Konsequenzialisten nach Mill, z. B. Sidgwick (1981, 382) oder Moore (1975, 110f.) und selbstverständlich auch Scheffler (1988, 1). Für diese Autoren ist es selbstverständlich, dass es sich so verhält. Unsere Rekonstruktion hat deutlich gemacht, dass es aus der Perspektive eines rational Handelnden keineswegs selbstverständlich ist, selbst wenn man den Standard für maximierende Rationalität akzeptiert. Dieser Punkt wird noch deutlicher, wenn wir uns im nächsten Abschnitt Kant zuwenden.

27 Insofern ist es irrelevant, die Tragfähigkeit von Mills Analogisierung von in der Metaphysik des Empirismus basal-deskriptiven Zugängen zu Gegenständen der Erfahrung (wie z. B. Töne, Schall oder Sichtbarem) mit normativ-affirmierten Gegenständen des Begehrens hinsichtlich ihrer Verbindlichkeit oder Legitimität weiterzuverfolgen. Vgl. zur Verteidigung dieser Analogisierung jedoch Müller (2003, 168ff.) oder Sayre-McCord (2001, 9-13), sowie Skorupski (1991, 286ff.). Wie wir im dritten Abschnitt zeigen wollen, sind alle Argumentationen, die das Gute im Sinne einer Setzung - sei diese auf empirischer Basis oder a priori eingeführt - vor Klärung des Verhältnisses des praktisch vernünftigen Akteurs zu dieser Setzung einführen, mit demselben methodologischen Problem, keine praktisch notwendige Verpflichtung durch das Gute begründen zu können, konfrontiert.
} 
eines Handelns aus Freiheit akzeptiert, die Frage stellen, warum es stets praktisch vernünftig sein sollte, aus Freiheit das Glück und nicht nur mein Glück zu realisieren. Warum also soll es immer gut sein, das Glück als ein Handlungsziel meinem Glück vorzuziehen? Wie also ließe sich ein Satz begründen, der nicht nur die Realisierung meines Glücks, sondern die Priorität einer Maximierung von Glück in der Welt, also gerade auch unparteiisches und altruistisches Handeln, verbindlich macht?28

Mills „Beweis“ kann die Notwendigkeit dieser Forderung vor allem deshalb nicht begründen, da der zugrunde liegende Begriff des Wollens in den Prämissen mehrdeutig ist und nicht hinreichend auf die Bedingungen einer praktischen Erkenntnis reflektiert wird. Mill übersieht die Zusatzprämisse eines Handelns aus Freiheit, die er seinem Beweis, da dieser eine praktische Forderung ableitet, hinzufügen müsste. In Prämisse 1 fokussiert Mill auf das Wollen im Sinne eines empirisch durch Beobachtung oder Introspektion nachweisbaren, zielgerichteten Verhaltens - eine wirkliche, durch Triebe ausgelöste Tätigkeit liegt vor (,,each person [...] desires his own happiness“"). In der 2. Prämisse und entsprechend auch in der Konklusion wird jedoch die empirisch-erklärende Perspektive auf das Verhalten vollständig verlassen..$^{29}$ Nun wird das Wollen als ein rationales Streben aufgefasst, bei dem durch Urteile bestimmte Zwecke als gut auszeichnet werden und das dabei der instrumentellen Rationalität in der Mittelwahl verpflichtet ist. Die Rede ist nun also von freien Handlungen und nicht mehr von bloßem Verhalten. Die Frage für einen frei Handelnden ist dann, warum das Urteil richtig ist, dass bestimmte Handlungen, die dazu geeignet scheinen, möglichst viel Glück in der Welt zu realisieren, notwendig gut seien, andere Urteile, die in einem Widerspruch zum Nützlichkeitsprinzip stehen, dagegen nicht?

Durch die erforderliche Zusatzprämisse eines Handelns aus Freiheit gerät Mills Beweisführung in ein Dilemma. Daher ist es nicht möglich, dass das Glück in praktischer Hinsicht ein letzter Zweck bzw. objektiv notwendig ein Gut ist. An dieser

${ }^{28}$ Die Problematik des Übergangs zum altruistischen Handeln wird seit Sidgwick immer wieder diskutiert (Sidgwick 1981, 387 ff.). Für eine Zusammenstellung der üblichen Kritikpunkte vgl. Sayre-McCord (2001, 6-9). Nach unserer Rekonstruktion erweist sich die fehlende Berücksichtigung der Zusatzprämisse des Handelns aus Freiheit (,praktische Lücke“) als das eigentliche Problem von Mills sog. Beweis. Sidgwick versucht, den Beweis zu retten, indem er die zweite Prämisse als normative Aussage auffasst, als zusätzliche Prämisse das Prinzip des unparteilichen Wohlwollens einführt und daraus dann gültig ableitet, dass das allgemeine Glück für die Gesamtheit der Menschen als Gut realisiert werden soll. Vgl. Sidgwick (1981, 387-389). Dazu Crisp (2017, S. 203 f.). Die Rekonstruktionen von Sayre-McCord und Müller bestätigen beide, wenn auch unfreiwillig, unser Ergebnis, dass Mill keine moralische Pflicht begründen kann. Nach Sayre-McCord ist das gar nicht Mills Beweisziel, denn es ginge ihm bloß darum, das allgemeine Glück als intrinsisches (d.h. nach unserer Terminologie „notwendiges“) Gut zu erweisen (Sayre-McCord 2001, 16). Diese Frage unterscheidet er von der Frage nach der moralisch richtigen Handlungsweise, was also jemand einen Grund gibt, etwas zu tun. Für diese Frage reicht nach Sayre-McCord der Verweis auf den selbstverständlichen Standard maximierender Rationalität bzw. auf die gelebte, auf Sanktionen beruhende Moral (Sayre-McCord 2001, 17). Diese Antwort ist aber unbefriedigend, denn zum einen geht es Mill sehr wohl darum, ein Kriterium für die moralische Bewertung zu begründen; zum anderen muss die Begründungsfrage von der Frage nach der Durchsetzung bzw. motivationalen Internalisierung eines konkreten, historisch-kontingenten Moralsystems unterschieden werden. Olaf Müller nimmt dagegen die Frage der Begründung moralischer Pflichten bei Mill ernst. Nach seiner Deutung kommt Mill beim ,Übergang in die Moral" aber nur zu einer schwachen, durch empirische Hypothesen plausibilisierten Begründung.

29 Die Problematik dieses Perspektivwechsels auf das Wollen - zum einen als Verhalten und zum anderen als Handeln - und das Erfordernis einer notwendigen Verbindung der beiden Momente diskutiert auch Georg Wilhelm Friedrich Hegel in den Grundlinien zur Philosophie des Rechts (1820/1986, § 125, 236). 
Stelle ist es wichtig festzuhalten, dass wir hier nicht das äußerliche Problem einer fehlenden moralischen Motivation des Handelnden oder einer Unempfindlichkeit gegenüber einer rationalen Beweisführung in Moralfragen adressieren wollen. Vielmehr liegt es in der Urteilsstruktur des praktischen Denkens über das Gute, dass es auch auf die Prämisse eines Handelns aus Freiheit festgelegt ist. Mill kann, wie wir rekonstruiert haben, durch seinen „Beweis“ zwar überzeugend darlegen, weshalb „das Glück“ theoretisch betrachtet als das notwendig Gute gedacht werden muss. Er übersieht jedoch die für Urteile über das Gute notwendige Strukturverbindung mit dem Vermögen der subjektiven Präferenzwahl eines Handelnden. Das führt dazu, dass der Beweis zwar als theoretisch plausibel und gültig rekonstruiert werden kann, aber aufgrund seiner ,,praktischen Lücke“ nicht zeigen kann, dass das Nützlichkeitsprinzip bei jeder Ausübung der Präferenzwahl befolgt werden muss.

Die Zusatzprämisse eines Handelns aus Freiheit können wir ausbuchstabieren, indem wir uns vergegenwärtigen, dass das Handeln in einem Urteil mit dem Prädikat gut (Affirmation) bzw. schlecht (Negation) besteht. Das erste Horn des Dilemmas entspricht der Antwortstrategie 1 (resp. 3). Mills Beweisführung wird vom Handelnden nachvollzogen und als gültig eingesehen. In einer Handlungssituation muss dann allerdings das Nützlichkeitsprinzip bzw. die entsprechend abgeleitete Handlung im Unterschied zu anderen Optionen affirmiert werden. Gemäß der Prämissen 1 und 2 des Mill'schen Beweises wird im Akt der Affirmation dann jedoch das Glück bzw. das Nützlichkeitsprinzip in die praktische Urteilsstruktur eingefügt. Dabei wird ,das Glück“ durch das Urteil des frei Handelnden jedoch relativiert auf je ,sein Glück“. „Das Glück“ erweist sich in der Urteilssequenz als ein nur relativ gutes (oder bei Negation ein nur relativ schlechtes) Mittel, um ,mein Glück“, das laut Prämisse 1 und 2 als gut beurteilt werden muss, zu realisieren.

Das zweite Horn des Dilemmas zeigt sich, wenn der Mill'sche Beweis für theoretisch überzeugend gehalten, der Bezug auf ein Handeln aus Freiheit aber geleugnet wird. Der Akteur kann, nachdem er den Beweis nachvollzogen und als gültig eingesehen hat, gegen das Nützlichkeitsprinzip und das Glück als das objektiv Gute in praktischer Hinsicht indifferent bleiben. Das liegt daran, dass eine logische Deduktion keinen Automatismus hinsichtlich der Präferenzwahl darstellt (schon allein deshalb, da überlegt werden muss, auf welche Weise dem womöglich überzeugenden Deduktionsergebnis im Handeln am besten entsprochen werden könnte). Der Handelnde kann sich dazu entscheiden, die theoretische Betrachtung des Deduktionsergebnisses durch Affirmation im Handeln umzusetzen. Diese Affirmation ist jedoch nicht notwendig. Es ist vielmehr kontingent, ob die logisch deduzierte Begründung affirmiert oder negiert wird. Das Nützlichkeitsprinzip könnte demnach nur dann ein notwendiges Prinzip sein, wenn es bloß theoretisch erwogen, also ohne die Zusatzprämisse eines Handelns aus Freiheit, und nicht auch als praktische Aufforderung, entsprechend zu handeln, gedacht wird.

In beiden Fällen ist es unmöglich, dass das Glück ein letzter Zweck bzw. objektiv gut ist; denn die möglichen Aktionen der Affirmation, Negation oder vorläufigen Indifferenz ,überschreiben“ das vermeintlich objektiv Gute und interpretieren es entweder als einen subjektiv als gut (oder als schlecht) beurteilten Zweck, oder als eine nur theoretisch interessante Vorstellung. 
Eine weiterführende Variante dieser dilemmatischen Situation entsteht daraus, dass Mill nicht nur zwei Verwendungsweisen von „Wollen“ (Verhalten und Handeln), sondern auch zwei Bedeutungen von Glück als einem letzten Zweck in seinem „Beweis“ integrieren und zusammenführen müsste. Wir können zum einen von einem auf Erfahrungsdaten basierenden, subjektiven Begriff des Glücks und zum anderen von einem rationalen, objektiven Begriff des Glücks sprechen. Um diesen beiden Bedeutungen gerecht zu werden, spricht Mill nach seinen Ausführungen zum „Beweis“ auch davon, dass es verschiedene, gleichsam selbstzweckhafte, subjektive Teile der objektiven Glücksvorstellung als eines Ganzen geben könne, z.B. Geld, das Gutsein als Tugend oder Liebe zur Tugend (Mill 2006, $113 \mathrm{ff}$.).

Mills Beweis und der deduktive Schluss auf die Formulierung des Nützlichkeitsprinzips wären auch in praktischer Hinsicht folgerichtig, wenn Glück in ausschließ $\beta$ lich objektiver Bedeutung durch Handeln realisiert werden könnte. Dem ist jedoch nicht so, wie Mill mit seinen Ausführungen zu subjektiv unterschiedlichen Teilen des Glücks und seiner Theorie von der empirischen Evidenz von Lust und Unlust als Indikatoren für Glück einräumen muss. Daher gilt, dass entweder die inhaltliche Setzung von Glück in subjektiver Bedeutung durch Abgleich mit den hierin Erfahrenen zu legitimieren ist (Mill 2006, 29f.), dann aber ist die Verbindlichkeit einer Realisierung von Glück in objektiver Bedeutung jeweils kontingent, da seine Bestimmung nur relativ zu einer selektiven Erfahrungsbasis und entsprechenden Entscheidung Geltung hätte. Oder es müsste gelten, dass, gemäß der Antwortstrategie 2, lediglich Glück in objektiver Bedeutung als letzter Zweck zu seiner Realisierung nötigen würde, dann wäre zwar der Beweis folgerichtig, aber dennoch nicht praktisch vernünftig, da, wie es Jean-Claude Wolf formuliert, die „Unmittelbarkeit von Evidenz [der Konklusion] und Wertung“ nicht nachgewiesen wurde. ${ }^{30}$ Es bleibt ungeklärt, wie eine objektive, gleichsam inhaltslose Vorstellung von einem empiristisch-hedonistisch verstandenen Glück ohne subjektive Interpretationszusätze auf Erfahrungsbasis im Handeln realisiert werden könnte.

Diese Konkretisierungsproblematik des „Beweises“ ist gerade auch dann virulent, wenn man, wie Mill es womöglich tat, dem „Beweis“ selbst keine große praktische Bedeutung beimessen will. Man müsste dann Mills Ausführungen hinsichtlich der Relevanz und Reichweite rationaler Argumentation für eine Veränderung der Praxis skeptischer verstehen und vielmehr eine ästhetisch-charakterliche Erziehung als eigentliches Ziel des utilitaristischen Projektes auffassen (Heydt 2006, 5, 58 ff., $153 \mathrm{f}$.). Umso mehr stellt sich dann jedoch die Frage, anhand welches Maßstabes der von Mill erhoffte ,moralische Fortschritt“ bemessen und woran ein staatliches Erziehungsprogramm ausgerichtet werden sollte? Und warum sollten wir Mills spezifischer Variante einer liberalistisch-humanistischen Weltanschauung folgen?

Mill scheint nun zwar nicht das oben beschriebene Dilemma (,praktische Lücke"), das sich aus der erforderlichen Zusatzprämisse eines Handelns aus Freiheit ergibt, gesehen zu haben, wohl aber das Problem der subjektiven Relativierung von Glück, wenn es durch einen Handelnden zu einem konkreten Handlungsziel gemacht wird. Im 5. Kapitel von Utilitarianism erklärt Mill nämlich, dass das Nützlichkeitsprinzip eine Unparteilichkeitsforderung enthalte und daher keine In-

30 Wolf $(2012,139)$. 
strumentalisierung von Handlungen, die dem allgemeinen Glück förderlich sind, auf die Realisierung des eigenen Glücks denkbar sei.

Die Forderung der Unparteilichkeit ,ergibt sich unmittelbar aus dem obersten Prinzip der Moral: Sie ist ein Teil des Nützlichkeitsprinzips [...]. Dieses Prinzip wäre nur eine Folge bedeutungsloser Worte, wenn nicht das Glück der Person bei gleichem Grad (und angemessener Berücksichtigung der Art) für genauso viel gelten würde wie das Glück jeder anderen. " ${ }^{\text {"31 }}$

Tatsächlich würde aus dem Existenznachweis von Glück in nur objektiver Bedeutung als letzter Zweck deduktiv nicht nur die Formulierung des Nützlichkeitsprinzips, sondern auch die Forderung der Unparteilichkeit bei der Realisierung von Glück folgen. Denn Glück in objektiver Bedeutung, so lässt sich Mill hier interpretieren, stellt eine Abstraktion von allen individuell-psychischen Besonderheiten der inhaltlichen Glücksvorstellungen dar. Aufgrund der begrifflichen Abstraktheit gelten Personen gleichermaßen als mögliche Träger von Glück, wobei begrifflich betrachtet keine Glücksvorstellung der anderen vorgezogenen werden kann - es steht kein Kriterium zur Differenzierung der Personen als potentiellen Trägern von Glück zur Verfügung. ${ }^{32}$ Mit der analytischen Rechtfertigung des Unparteilichkeitsgebots scheint Mill die nur egoistisch-parteiliche Orientierung auf mein Glück widerlegen zu können. Da Glück jedoch immer auch auf Erfahrungsbasis bestimmt werden muss, denn sonst könnte es keine Relevanz als wirklicher Zweck bestimmter Handlungen haben, ist das Unparteilichkeitsgebot hinfällig. Eine spezifische Glücksvorstellung, die z.B. nur auf Kosten der Möglichkeit zur Realisierung vieler, kontradiktorischer Glücksvorstellungen realisierbar wäre, wird dann zwar als mögliche berücksichtigt, aber ist nach dem Nützlichkeitsprinzip relativ schlecht und sollte nicht realisiert oder unterstützt werden.

Die Unparteilichkeitsforderung ist daher entweder handlungspraktisch irrelevant, weil sie nur das Glück in objektiver Bedeutung betrifft, oder sie ist, was ihre praktische Verbindlichkeit betrifft, als ein Mittel relativiert und somit aufgehoben durch jeweils erfahrungsbasierte Bestimmungen von Glück in subjektiver Bedeutung. Wir sind damit wieder bei der oben genannten Problematik von Antwortstrategie (1.) angekommen; der Dezisionismus in der Verbindlichkeit des Nützlichkeitsprinzips wurde nicht überwunden. ${ }^{33}$

31 Vgl. Mill (2006, 185). Systematisch stringenter führt Sidgwick das Prinzip der Unparteilichkeit ein (Sidgwick 1981, 379-384). Seitdem fehlt die Berufung darauf in keiner Lehrbuchdefinition des Konsequenzialismus.

32 Für die quantitative Verteilung endlicher Glückseinheiten in der Welt bleibt freilich das Problem einer mit dem Nützlichkeitsprinzip zwar vereinbaren, aber ungleichen Verteilung bestehen: 100 Glückseinheiten lassen sich auf 100 potenzielle Träger verteilen oder auf nur einen, das Additionsverhältnis der Summe ist letztlich beliebig. Diesen kalkulatorischen Problemen des utilitaristischen Ansatzes wollen wir hier jedoch nicht weiter nachgehen. Vgl. dazu Rawls (1988, 45).

33 Dass dieser Dezisionismus mit den Mitteln von Mills empiristischer Methodologie nicht überwunden werden kann, verdeutlichen auch die Ausführungen von Olaf Müller (2003, 181-189). So kann Mill zwar im ersten Schritt seines Arguments (d.h. nach unserer Rekonstruktion im Übergang von Prämisse 2 zur Konklusion) zu einer objektiven „Beschreibung der kollektiven Werthaltung aller Menschen vordringen“. Dieser Schritt ist allerdings, wie Müller selbst betont, nur um den Preis äußerst voraussetzungsreicher psychologischer Zusatzprämissen als vermeintlich gesicherte, empirische Tatsachenaussagen möglich. Dem- 
Bevor wir zum nächsten Abschnitt übergehen, sei ausdrücklich betont, dass unser Argument nicht mit der Diagnose eines Sein-Sollens-Fehlschlusses oder eines naturalistischen Fehlschlusses verwechselt werden darf. Nach unserer Kritik begeht Mill nicht den Fehler, aus faktischen Wünschen der Menschen normative Forderungen ableiten zu wollen. ${ }^{34}$ Unser Argument hat zwei Hauptschritte und beruht auf einer Präsuppositionsanalyse, die den Standpunkt einer praktisch vernünftig überlegenden und handelnden Person einnimmt. Von diesem Standpunkt aus lässt sich zunächst in reflexiver Rekonstruktion zeigen, dass der Schluss auf ein notwendiges Gut gültig ist. In einem zweiten Schritt wird gezeigt, dass der Beweis eine praktische Lücke aufweist, die in ein Dilemma führt. Diese Lücke wird deutlich, wenn man die vom Standpunkt eines praktisch reflektierenden Subjekts notwendige Prämisse eines Handelns aus Freiheit berücksichtigt. Aus dem Dilemma folgt, dass es Mill nicht gelingt, die im weiten Sinne logische Schlussfolgerung auf ein Prinzip des Guten (Glück als letzter Zweck) auf praktisch vernünftige Weise mit der Verpflichtung zur Realisierung durch ein Handeln aus Freiheit zu verbinden. Die Verbindung von Handeln als freie Zwecksetzung und Mittelwahl mit der Verbindlichkeit, dem Prinzip des Guten entsprechend zu handeln, ist letztlich kontingent, da sie begrifflich an die subjektive Präferenzwahl des Akteurs gebunden bleibt.

Im folgenden Abschnitt wollen wir auf der Basis einer weiteren, methodologischen Kritik des „Beweises“ nicht nur zeigen, dass das eingangs von Scheffler formulierte deontologische Paradox unzutreffend ist und nicht besteht, sondern auch, dass ganz im Gegenteil sich Schefflers utilitaristische Position (genauso wie die Position Mills) vor das Problem gestellt sieht, überhaupt keine normativen Aussagen als praktisch vernünftig ableiten zu können. Das liegt vor allem daran, dass Mill den Begriff ,gut“ weitgehend unkritisch in Analogie zur theoretischen Erkenntnis des Wahren verwendet und nicht auf die eigentümlichen Erkenntnisbedingungen des Guten reflektiert.

nach sind Menschen nicht nur so konstituiert, dass sie Glück aus der Beförderung des Glücks anderer ziehen können, sondern, ,dass sie das ihnen wertvollste Glück nur aus der Beförderung des Glücks anderer ziehen können“ (Müller 2003, 181). Mit dem zweiten, uns hier besonders interessierenden Schritt, den Müller als „Übergang in die Moral“ (Müller 2003, $183 \mathrm{ff}$.) bezeichnet, lässt sich auf dem empiristischen Weg nur eine sehr schwache normative Forderung begründen, wonach der letzte Zweck menschlichen Handelns das allgemeine Glück sein sollte. Die beiden dafür erforderlichen Zusatzannahmen, wonach es Güter gibt, ,die um ihrer selbst willen angestrebt werden sollten“ und dass „,der Standard für diese Güter bei uns Menschen liegt und nicht irgendwo jenseits der Menschheit“, sind gewiss harmlos, erlauben aber keine Begründung der notwendigen praktischen Verbindung. Ob der einzelne Akteur das vom Maximierungsprinzip geforderte altruistische Handeln als verbindlich anerkennt, bleibt wie bei Mill kontingent. Müllers abschließendes Zugeständnis, dass „Mills Übergang in die Moral nicht logisch erzwungen werden kann“ (Müller 2003, 189), muss dann als Bestätigung unseres Ergebnisses verstanden werden, dass es keinen praktisch-vernünftigen Grund gibt, von der egoistischen Verfolgung des Glücks überzugehen zur Pflicht, das Glück aller zu befördern.

34 Ein Einwand dieser Art ist bekanntlich von George Edward Moore formuliert worden. Moore spricht allerdings von einem ,,naturalistischen Fehlschluss“ und entwickelt sein Argument im Zusammenhang mit der Frage nach der Definierbarkeit von ,gut“ als eines nicht-natürlichen, einfachen Prädikats: Aus der Tatsache, dass bestimmte natürliche Eigenschaften als gut bezeichnet werden können, wird, so Moores Argument, zu Unrecht auf die Synonymie von ,gut“ mit diesen natürlichen Eigenschaften geschlossen. Wie William Frankena herausgearbeitet hat, ist es daher wohl angemessener von einem ,Definitions-Fehlschluss“ (Frankena 2016, 57) zu sprechen. Vgl. Moore (1970, 35-41, zu Mill: 108-120). 


\section{Das „Paradoxon der Methode in einer Kritik der praktischen Vernunft" - oder: warum die konsequenzialistische Argumentation scheitern muss}

Mit der Frage nach der Erkennbarkeit des Guten hat sich dagegen Immanuel Kant konfrontiert. Kant problematisiert den Begriff einer praktischen Erkenntnis als die metaethische Aufgabe, wie eine solche Erkenntnis, die mit dem Prädikat ,gut“ operiert, als vernünftige Einsicht in eine logische Notwendigkeit und zugleich als eine Festlegung des Handelns aus Freiheit möglich ist. Zu klären ist also, wie sich der frei Handelnde, der bei Mills Beweisführung ohne Explikation mitvorausgesetzt wird, beim praktischen Erkennen zu den Gründen, etwas tun zu müssen, verhält. Im Gegensatz zu Mill diskutiert Kant die Begründungsproblematik insgesamt als ein Problem des Wissens vom Guten (also nicht als eines der Motivation bzw. als theoretische Anleitung zu einer nachträglichen praktischen Präferenzwahl). ${ }^{35}$

Kant argumentiert in der Kritik der praktischen Vernunft im Anschluss an die Ausführungen zur Analytik der reinen praktischen Vernunft für die Auflösung eines vermeintlichen „Paradoxon der Methode“, das sich aufgrund einer deontologischen Umgangsweise mit dem Begriff des Guten scheinbar ergibt. Diese methodologische Auseinandersetzung ist bisher noch wenig beachtet worden. ${ }^{36}$ Worin besteht nun das von Kant konstatierte vermeintliche „Paradoxon“?

Hier ist nun der Ort, das Paradoxon der Methode in einer Kritik der praktischen Vernunft zu erklären: dass nämlich der Begriff des Guten und Bösen nicht vor dem moralischen Gesetze (dem er dem Anschein nach sogar zum Grunde gelegt werden müsste), sondern nur (wie hier auch geschieht) nach demselben und durch dasselbe bestimmt werden müsse. (KpV, AA V, 62)

Wie im vorherigen Abschnitt gezeigt, verhält sich Mills Beweisverfahren gegenteilig zur im Zitat explizierten Methode einer Kritik der praktischen Vernunft. Mill hatte in einem ersten Schritt durch eine Schlussfolgerung den Begriff des Guten bestimmt: „Glück“ ist das objektiv notwendige Gut. Alles andere sei nur relativ dazu gut, nämlich als mögliches Mittel zur Realisierung von Glück. In einem zweiten Schritt dient die Konklusion der Schlussfolgerung als Prämisse zur analytischen Ableitung des Nützlichkeitsprinzips. Nach Mill ist das ,,principle of utility“ (Mill 2006, 104) das einzig denkbare Moralprinzip, um gut und schlecht unterscheiden zu können. ${ }^{37}$

Kant stellt dagegen den Begriff des Gesetzes ins Zentrum seiner Überlegungen zur praktischen Philosophie, um zu klären, wie eine praktische Notwendigkeit als eine Forderung von einem freien Subjekt überhaupt erkannt werden kann. So wird in der Vorrede der Grundlegung zur Metaphysik der Sitten ein „Gesetz“ definiert

\footnotetext{
35 Vgl. dazu ausführlich Engstrom (2009) und Richter (2020).

36 Vgl. allerdings Sensen (2013, $69 \mathrm{ff}, 75 \mathrm{f}$.).

37 Mill spricht z. B. vom „utilitarian standard“ und dessen ,formula“ (Mill 2006, 18) bzw. vom ,standard of morality“ $(2006,38)$ als ,,criterion of right and wrong“ $(2006,6)$ sowie von einem höchsten ,principle“ (2006, 104).
} 
als Satz, der „,absolute Notwendigkeit bei sich führ[t]““.38 In der Kritik der praktischen Vernunft führt Kant dagegen, wie Michael Wolff herausgearbeitet hat, den Begriff eines praktischen Gesetzes im Paragraph 1 der Analytik - gleichsam more geometrico - per Deklaration als einen möglichen Begriff ein (Wolff 2009, 511-549, $516 \mathrm{ff}$.): Ein praktisches Gesetz sei eine ,allgemeine Bestimmung des Willens“, die als objektiv, „d. i. für den Willen jedes vernünftigen Wesens gültig erkannt wird“ (KpV, AA V, 19). ${ }^{39}$ Die Adjektive „moralisch“ oder ,praktisch“, die in Verbindung mit dem Gesetzesbegriff auftreten, können synonym verstanden werden, insofern sie eine Rede über die „Gesetze der Freiheit“"40 bzw. eine begriffliche Auseinandersetzung mit „gut“ und „böse“ anzeigen. ${ }^{41}$ Das Spektrum der Beurteilung, ob etwas in diesen Bereich der praktischen Vernunft fällt (d.h. „Objekt einer möglichen Wirkung durch Freiheit“ ist), ${ }^{42}$ ist einerseits durch naturgesetzliche Modalitäten (ist es naturgesetzlich möglich oder unmöglich, die Handlung durchzuführen, durch die der Gegenstand herbeigeführt werden soll?), andererseits durch „,moralische“ Modalitäten eingeschränkt. Maßgeblich für letztere sind die praktischen Modaloperatoren „müssen“ bzw. „sollen“ (unmöglich/notwendig) oder „,erlaubt“ (möglich). ${ }^{43}$ Die Überprüfung, ob etwas getan werden muss, ist, nach Kant, Sache der praktischen Vernunft, dem Vermögen, einzelne Handlungen aus Prinzipien abzuleiten (GMS, AA IV, 412). Auf diese Weise wird erkannt, ob eine Handlung gut ist oder nicht.

„Gesetz“ steht in Kants Einlassung zum „Paradoxon der Methode“ also für einen Satz, der sich auf Freiheit bezieht und anzeigt, was getan werden muss. Aufgrund der erforderlichen Voraussetzung der freien Präferenzwahl und zur Abgrenzung von naturgesetzlich erklärbarem Zwang sollten wir jedoch präziser sagen: „Gesetz“ steht

${ }^{38}$ GMS, AA IV, 389: „Jedermann muss eingestehen, dass ein Gesetz, wenn es moralisch, d. i. als Grund einer Verbindlichkeit, gelten soll, absolute Notwendigkeit bei sich führen müsse.“

${ }^{39}$ Durch indirekte Beweise, die mit vollständig disjunktiven Begriffen operieren, entwickelt Kant dann analytisch die Formel eines möglichen praktischen Gesetzes. Die Möglichkeit dieser Formel - über ihre bloße Denkbarkeit hinaus - wird schließlich durch das Postulat ihrer Ausführbarkeit in Paragraph 7 der Analytik als wirkliche Nötigung erwiesen (Wolff 2009, $522 \mathrm{ff}$.). Die Rechtfertigung der als Willensbestimmung einzig möglichen Formel eines praktischen Gesetzes zeigt zugleich, was die einzig denkbare Bestimmung von „gut“ als dem Prädikat einer Erkenntnis des praktisch Notwendigen sein kann.

40 GMS, AA IV, 389: Die „Gesetze der Freiheit“ sind solche, ,nach denen alles geschehen soll, aber doch auch mit Erwägung der Bedingungen, unter denen es öfters nicht geschieht." Der Nachsatz zur Charakterisierung der Gesetze der Freiheit verdient besondere Beachtung: Wenn nämlich die durch die Erkenntnis eines Sollens geforderte Tat nicht nur „öfters“ geschehen, sondern immer geschehen würde, dann handelte es sich bei der erkannten Notwendigkeit um kein Gesetz der Freiheit, sondern um ein Gesetz der Natur. Mit diesen grundbegrifflichen Unterscheidungen in der Vorrede der Grundlegung zur Metaphysik der Sitten eröffnet Kant also die Problematik, unter welchen Bedingungen eine Erkenntnis von praktischer Notwendigkeit operieren können muss.

${ }^{41}$ Der Ausdruck ,moralisch“ bezeichnet hier ganz allgemein, dasjenige, was mit der Freiheit des Willens in Beziehung steht. Zu dieser Bedeutung von „moralisch“ vgl. die instruktiven Ausführungen bei Kobusch $(2016,19)$.

${ }^{42} \mathrm{KpV}$, AA V, 57: „Unter einem Begriff der praktischen Vernunft verstehe ich die Vorstellung eines Objekts einer möglichen Wirkung durch Freiheit.“ Vgl. ebd.: „Ein Gegenstand der praktischen Erkenntnis, als einer solchen, zu sein, bedeutet also nur die Beziehung des Willens auf die Handlung, dadurch er, oder sein Gegenteil, wirklich gemacht würde".

43 „Was wir gut nennen, muss in jedes vernünftigen Menschen Urteil ein Gegenstand des Begehrungsvermögens sein“ (KpV, AA V, 60 f.). Der Ausdruck ,gut“ bezeichnet einen Gegenstand der praktischen Vernunft, sofern wir seine Existenz „,wollen dürfen“ (KpV, AA V, 58). 
für das, was aufgrund von Einsicht in Gründe getan werden muss. Wie wir oben gesehen haben, kann Mill nun gerade nicht nachweisen, warum das als notwendig gut Erkannte auch realisiert werden muss. Das Dilemma der ,praktischen Lücke“ in Mills Beweis zeigt, dass das Nützlichkeitsprinzip unter keinen Umständen eine praktisch notwendige Forderung sein und auch kein Prinzip zur Ableitung von notwendig zu präferierenden Handlungen sein kann.

Woran liegt das? Wenn wir Kants Überlegungen folgen, ließe sich die Problematik des Mill'schen Beweis' nur vermeiden, wenn - paradoxer Weise - das Ableitungsverhältnis der Begriffe des Guten und der gesetzlichen Forderung, dieses Gute zu realisieren, umgekehrt würde. Der Begriff des Guten dürfte nicht dem Gesetz zugrunde gelegt werden, sondern müsste ,nach demselben und durch dasselbe“ bestimmt werden. Ersteres geschieht jedoch in Mills Beweis: „Das Glück“ wird als das einzig denkbare objektiv Gute bestimmt und daraus wird die Formel des Nützlichkeitsprinzips abgeleitet, aus dem wiederum die Differenzierung von guten und schlechten Handlungen abgeleitet werden soll.

Kant geht anders als Mill von einer metaethischen Überlegung über den Bereich der Erkenntnis des Guten aus. Anders als Mill bestimmt er nicht zunächst etwas, das eigentlich und unter allen Umständen gut ist (bei Mill: das Glück) und leitet daraus ein Moralprinzip ab (bei Mill: das Nützlichkeitsprinzip), das angibt, wie der als notwendig gut bestimmte Zielzustand erreicht wird. ${ }^{44}$ Vielmehr geht Kant von einer formalen Bestimmung des Urteilsprädikats ,ist gut“ aus, das bei jeder Präferenzwahl verwendet werden muss. „Gut“ ist in dieser formalen Bestimmung das, was als praktisch notwendig erkannt wird, d.h., von der im Urteil als gut erkannten Handlung ist es nicht möglich, dass sie nicht getan wird. ${ }^{45}$

Angesichts einer praktischen Notwendigkeit muss es, so können wir mit Kant feststellen, immer auch möglich sein, dass die Forderung, so müsse es getan werden, aus Freiheit affirmiert oder negiert wird. Praktische Notwendigkeit muss von naturgesetzlicher Notwendigkeit begrifflich unterscheidbar sein. Andernfalls stünde das Gesetz (Kants Synonym für absolute Notwendigkeit) nicht in einem praktischen Verhältnis der Nötigung zur praktischen Vernunft des Akteurs, sondern im Sinne einer naturgesetzlichen Notwendigkeit, die bestimmte Verhaltensweisen determinieren würde. Dann aber wäre das Gesetz keine praktische, sondern eine theoretische Erkenntnis des Guten, die für eine Begründung der richtigen Präferenzwahl irrelevant wäre (s. oben: zweites Horn des Dilemmas).

Jede Argumentation, die etwas als gut auszeichnen (bei Mill: das Glück) und dessen Verwirklichung mit Notwendigkeit fordern soll, steht damit vor dem Problem, zwei zunächst widersprüchliche Aussagen vereinbaren zu müssen. Aufgrund des Vermögens der Präferenzwahl (die von Mill übersehene Zusatzprämisse des Handelns aus Freiheit), das die subjektiv-normative Instanz der Realisierung von

\footnotetext{
44 Kant bezeichnet ein derartig inhaltlich bestimmtes Gut auch als „Begriff von einem Gegenstande (als einem guten)“ (KpV, AA V, 63), daher scheint uns die Rede von einem vorgestellten ,Zielzustand“ passend zu sein. Ein Zustand (als Gegenstand einer Vorstellung) erscheint als gut und soll durch Handeln realisiert werden.

45 GMS, AA IV, 412: „Der Wille ist ein Vermögen, nur dasjenige zu wählen, was die Vernunft unabhängig von der Neigung als praktisch notwendig, d. i. als gut, erkennt.“
} 
Handlungen durch Begründungen darstellt, muss gelten (1.): „Es ist kontingent, was getan werden soll. "46 Durch diesen Satz lässt sich das Vermögen der Präferenzwahl charakterisieren, die zwar durch praktische Erkenntnis zur Realisierung des Guten genötigt, nicht aber notwendig bzw. gleichsam automatisch durch eine externe Autorität zum Handeln bestimmt wird. Jedoch muss, aufgrund der avisierten Objektivität des Begriffs des Guten, das Gute als etwas vorgestellt werden, das notwendig durch das Handeln herbeigeführt werden soll, und somit zugleich die kontradiktorische Aussage gelten (2.): „Es ist nicht kontingent, was getan werden soll (vielmehr muss das Gute realisiert werden).“

Weil der Begriff des Guten von Mill im Vorhinein und ohne Bezug zum Vermögen der Präferenzwahl eingeführt wurde, ergibt sich für sein Argument misslicher Weise die Struktur zweier strukturell isolierter Entitäten: Das Gute ist für sich durch Notwendigkeit, dagegen das Vermögen der Präferenzwahl durch Kontingenz hinsichtlich seiner Aktualisierung bestimmt. In Mills Beweis ist das Verhältnis des Guten und der Präferenzwahl aufgrund der logischen Reihenfolge der Einführung der Begriffe notwendig kontingent. Es kann sein, dass das Vermögen der Präferenzwahl genauso wie das Gute, das seine Realisierung mit Notwendigkeit fordert, bestimmt ist, aber dies muss nicht so sein. ${ }^{47}$ Somit ist die Übereinstimmung der Präferenzwahl mit dem Guten bei Mill immer nur beiläufig - wir hatten das oben als das Dilemma der praktischen Lücke rekonstruiert. Aufgrund der Möglichkeit der freien Präferenzwahl ist die Affirmation des Guten etwas, wie Kant diagnostiziert, das zur Einsicht in die praktische Notwendigkeit des Guten logisch nachträglich hinzukommt. Das Gute erweist sich dadurch als nur relativ praktisch notwendig, nämlich unter der Bedingung einer affirmativen Zwecksetzung durch Präferenzwahl. Man könnte auch sagen, nicht das Gute bestimmt hier die Präferenzwahl, sondern die Präferenzwahl bestimmt das Gute als ein relativ gutes Mittel zur Realisierung eines anderweitig

46 Davon zu unterscheiden ist die mit dieser praktisch-normativen Aussage verbundene theoretische Aussage, dass es - zumindest in subjektiver Perspektive - kontingent ist, was geschieht und was geschehen wird. Denn das, ,was geschehen wird“ kann nur auf Basis bisheriger Erfahrung und verfügbarer Information extrapoliert werden. In dieser praktischen Perspektive kann ein absoluter Determinismus, insofern ein Interesse am Handeln und der Beeinflussung von Geschehen besteht, nicht vertreten werden.

47 Mill argumentiert also für einen Zielzustand, der notwendig gut sei (das Glück). Das gelingt ihm auch. Nur muss er dann auch behaupten, dass eigentlich nichts anderes als dieser Zielzustand ,gut“ ist und u. a. die Zusatzprämisse des Handelns aus Freiheit ignorieren. Kant drückt das wie folgt aus: „Gesetzt, wir wollten nun vom Begriffe des Guten anfangen, um davon die Gesetze des Willens abzuleiten, so würde dieser Begriff von einem Gegenstande (als einem guten) zugleich diesen als den einzigen Bestimmungsgrund des Willens angeben." (KpV, AA V, 63) Da aber der als notwendig gut gesetzte Zielzustand in praktischer Hinsicht auch durch das Urteil eines Handelnden erkannt und realisiert werden muss, ergibt sich eine Verdopplung der Begriffe des Guten. Denn im Verhältnis zum denkenden und handelnden Subjekt könnte, so Kant, dann ,der Probierstein des Guten oder Bösen in nichts anders, als in der Übereinstimmung des Gegenstandes mit unserem Gefühle der Lust oder Unlust gesetzt werden." (KpV, AA V, 63) Die Rede von Lust und Unlust ist hier nicht empirisch-psychologisch zu verstehen, sondern Ausdruck der normativen Instanz der Präferenzwahl, die begrifflich betrachtet zum Guten, wenn es als ,ein Gegenstand“ eingeführt wird, immer in einem Verhältnis der Negation (Unlust) oder Affirmation (Lust) stehen können muss. Die Verdopplung des Guten besteht darin, dass etwas unabhängig vom Handelnden als notwendig gut gesetzt wird, dieses als zu erreichender Zielzustand aber nur durch ein affirmatives Urteil eines Handelnden praktisch gut ist. Die eigentliche normative Instanz ist dann aber nicht das Gute (als ein Gegenstand), sondern das Vermögen der Präferenzwahl des Handelnden, der zum objektiv Guten - je nach Lust und Unlust - ein kontingentes Verhältnis hat. Der Handelnde kann diesen Zielzustand erstreben, er muss es jedoch nicht. 
als gut bestimmten Zweckes. Damit ist es jedoch ausgeschlossen, dass die Aktivität des Herbeiführens irgendeines Zielzustandes (bei Mill: das Glück), der als objektiv gut bezeichnet wird, objektiv gut ist. ${ }^{48}$ Die von uns in Mills Beweis diagnostizierte praktische Lücke verweist auf eine logische Paradoxie konsequenzialistischer Argumentationen: Der Zielzustand gilt als praktisch notwendig; es soll nicht möglich sein, dass die Handlungen zur Herbeiführung dieses Zustandes nicht getan werden. Es besteht also die Forderung, diese unter allen Umständen zu tun. Sobald aber gefragt wird, warum das getan werden muss, müsste wiederum auf andere, entferntere Zielzustände verwiesen werden, die womöglich subjektiv präferiert werden. Dann aber ist die Herbeiführung des praktisch notwendigen Zielzustandes (in Mills Terminologie: der letzte Zweck) nicht praktisch notwendig, sondern ein austauschbares Mittel, um einen anderen Zielzustand zu erreichen.

Kant weist darauf hin, dass das Verhältnis einer praktisch notwendigen Verbindung von Präferenzwahl und dem Begriff des Guten nicht denkbar ist, wenn deren Notwendigkeit als praktische von einer kontingenten Affirmation abhängig ist. Das liegt daran, dass die Affirmation oder Negation durch das Vermögen der Präferenzwahl wiederum selbst mögliche Handlungen (mit Blick auf Zielzustände) sind und insofern mit dem Prädikat ,gut“ operieren müssen. Das objektive Gute wird dann, da dessen Affirmation selbst eine Handlung darstellt, durch einen subjektiv gesetzten Begriff des Guten relativiert.

Die Prädikation mit „gut“ käme damit doppelt vor: logisch primär als Gegenstand der Erkenntnis und sodann als Affirmation bei der Präferenzwahl. Durch das Erfordernis der Affirmation wird die Gültigkeit des objektiv Guten auf die normative Instanz der Willkür relativiert. Die Möglichkeit von Affirmation (oder Negation) des objektiv Guten darf jedoch nicht ausgeschlossen werden, da die erkannte Notwendigkeit andernfalls als eine naturgesetzliche gelten müsste. Daraus folgt, dass das Problem, warum das als gut Erkannte auch realisiert werden muss, im Rahmen der von uns diskutierten konsequenzialistischen Strategie nicht gelöst werden kann. Vielmehr, und das kann als Kern der deontologischen Argumentationsstrategie angesehen werden, muss das, was als notwendig gut erkannt werden kann, durch ein reflexives Urteil des Handelnden, oder anders gesagt: im Sinne einer Präsuppositionsanalyse des Operierens mit dem Prädikat gut bestimmt werden. ${ }^{49}$

Aus Kants Einsicht, dass die Möglichkeit einer notwendigen Verbindung des objektiv Guten und des subjektiven Vermögens der freien Präferenzwahl vor einer Bestimmung des Guten als eines Zielzustandes geklärt werden muss, lassen sich drei methodologische Gesichtspunkte für moralphilosophische Überlegungen im Allgemeinen festhalten. Diese werden in der konsequenzialistischen Konzeption der praktischen Vernunft bei Mill und Scheffler nicht berücksichtigt.

\footnotetext{
48 Wie Kant es ausdrückt, ist durch die Bestimmung des Guten als eines Zielzustandes ,geradezu die Möglichkeit praktischer Gesetze a priori ausgeschlossen: weil man vorher nötig zu finden meinte, einen Gegenstand für den Willen auszufinden, davon der Begriff als eines Guten den allgemeinen, obzwar empirischen Bestimmungsgrund des Willens ausmachen müsse." (KpV, AA V, 63)

49 Vgl. Bambauer (2019, S. 9 f., 66, 350-360). Vgl. Steigleder (2002, 33; 23-58). Vgl. Grundmann (1994, 296-310). Im Anhang stellen wir eine Rekonstruktion der Kantischen Argumentation in GMS und KpV mit dem formalen Begriff des Guten als Erkenntnis des praktisch Notwendigen dar.
} 
(A.) Die Prädikate „gut“ und „schlecht“ sind praktische Begriffe, d.h., sie betreffen Erkenntnisse als mögliche Wirkungen von Freiheit. Die Bestimmung dieser Begriffe kann also nicht isoliert vom Handeln und dessen normativer Instanz der Präferenzwahl durchgeführt werden. ${ }^{50}$ Jeder Versuch, den Begriff ,gut“ einzuführen, muss diesen als mögliche Bestimmung durch die freie Präferenzwahl betrachten, die eine besondere Erkenntnis des Guten auch negieren können muss.

(B.) Das objektive Gute kann nicht als isoliertes Objekt mit unklarem Verhältnis zum Erkenntnissubjekt eingeführt werden. Als Objekt wäre seine Realisierung, da es zugleich Gegenstand der praktischen Erkenntnis und einer möglichen subjektiven Affirmation sein muss, immer nur kontingenter Weise verbindlich, nicht aber notwendig. Gemäß Kants Ausführungen zum „Paradoxon der Methode“ ist es daher erforderlich, mit der Frage zu beginnen, unter welchen Bedingungen eine Erkenntnis des praktisch Notwendigen möglich sein kann, ohne dabei das Vermögen der Präferenzwahl aufzuheben. Dies ist, wie wir herausgestellt haben, aber nur dann möglich, wenn sich ein praktisches Gesetz konzipieren lässt. Dieses Gesetz kann jedoch, genauso wie der Begriff des Guten, nicht als Objekt der Erkenntnis eingeführt werden. Da der Gesetzescharakter durch Relativierung auf eine erforderliche Affirmation durch die subjektive Prädikation als ,gut“ aufgehoben würde.

(C.) Um die weiter oben eingeführten Sätze (1.) und (2.) ${ }^{51}$ ohne Widerspruch verbinden zu können, muss diese Verbindung in einem affirmativen Urteil, das vom Handelnden nicht unterlassen werden kann, bestehen. Dabei darf zudem kein Widerspruch zu Satz (1.) auftreten. Satz (2.), also ein Begriff des objektiv Guten, wäre dann eine Folgerung aus der Möglichkeit einer derartigen notwendigen Affirmation. Aufgrund der methodischen Gesichtspunkte (A.) und (B.) muss eine solche, gleichsam notwendige Affirmation reflexiv als Nachweis einer höherstufigen Affirmation erfolgen (in Gestalt einer Präsuppositionsanalyse), die die objektstufige Affirmationen oder Negationen von möglichen Handlungsweisen allererst durchführbar macht.

\section{Fazit: Die Auflösung des ,deontologischen Paradox“ durch eine Reflexion auf die Erkenntnisbedingungen des Guten}

Schefflers Argumentation zielte auf den Nachweis, dass unbedingte Pflichten - oder in seiner Terminologie: agent centred restrictions - nicht begründet werden können, wenn man vom allgemein akzeptierten Standard der maximierenden Rationalität und einer damit verbundenen konsequenzialistischen Teleologie ausgeht. Unbedingte Pflichten müssen dann paradox erscheinen. Unsere an Kant angelehnte methodische Reflexion hat dagegen gezeigt, dass Scheffler, wenn auch aus anderen Gründen,

\footnotetext{
50 Hier fällt eine Parallele zu Aristoteles' Konzept von Praxis und seiner Kritik an essentialistischen Auffassungen der Ideenlehre auf. Auch nach Aristoteles ist ,gut“ ein wesentlich praktischer Begriff, (EN I, 4, 1096b 32-35): „Auch wenn das Gute existiert, das eines ist und allgemein ausgesagt wird, oder das abgetrennt und an und für sich besteht, so ist es doch klar, dass dieses Gute für den Menschen weder zu verwirklichen noch zu erwerben ist. Nun ist es aber ein solches, das wir suchen.“

51 „Es ist kontingent, was getan werden soll“ (1.) (freie Willkür bzw. das Vermögen der Präferenzwahl) und (2.) „Es ist nicht kontingent, was getan werden soll (vielmehr muss das Gute realisiert werden)“ (das objektiv Gute).
} 
hierbei zugestimmt werden kann. Strikte Pflichten lassen sich in der Tat auf der Grundlage der konsequenzialistischen Konzeption von Rationalität nicht begründen. Es genügt, sich klar zu machen, dass der Begriff des Guten als ein wesentlich praktischer Begriff mit dem Begriff der praktischen Notwendigkeit im Erkenntnisakt verbindbar sein muss. Nur das im praktischen Sinne als notwendig Erkennbare kann als ,gut“" gelten und muss realisiert werden.

Konsequenzialistische Theorien können jedoch keinen Begriff des Guten entwickeln, der sowohl die Erkenntnis einer logischen Notwendigkeit als auch einer praktischen Relevanz zugleich zum Ausdruck bringen kann. Sie setzen vielmehr den Begriff des Guten als einen letzten Zweck voraus, ohne ihn begrifflich mit der praktischen Vernunft des Handelnden verbinden zu können. Die begriffliche Möglichkeit dieser Verbindung muss aber begründet werden, weil nur dann verständlich wird, warum der Handelnde sich dem Prinzip, das aus dem Begriff des Guten abgeleitet wird, aufgrund der Einsicht in eine Notwendigkeit unterwerfen soll und das Gute realisieren muss.

Wie wir rekonstruiert haben, weist der Mill'sche „Beweis“ keinen Deduktionsfehler auf. Das Kernproblem liegt jedoch in einer praktischen Lücke des Beweisverfahrens. Sie besteht darin, dass Mill die Zusatzprämisse des Handelns aus Freiheit nicht berücksichtigt. Diese Prämisse ergibt sich aus der Struktur des praktischen Denkens, die auch vom Konzept maximierender Rationalität anerkannt wird. Insofern ist es gerade im Namen der praktischen Vernunft erforderlich, diese Prämisse zu berücksichtigen. Es handelt sich um eine Prämisse, die in der reflexiven Einstellung eines praktisch überlegenden Subjekts eingeholt wird. Insofern weist der Beweis tatsächlich eine praktische Lücke auf und keinen bloß deduktionslogischen Defekt. Wir haben diese Lücke als ein Dilemma dargestellt, in das Mill gerät, sobald man die notwendige Zusatzprämisse eines freien Handelns mitdenkt. Es zeigt sich dann, dass die Schlussfolgerung des Beweises keine notwendige Verbindung im Sinne einer Verpflichtung, die zugleich praktisch wirksam ist, herstellen kann. Diese praktische Lücke in Mills Beweis ist einem Defizit an methodologischer Reflexion auf die Bedingungen der Erkennbarkeit des Guten geschuldet. Das damit angezeigte Defizit betrifft nun nicht nur Mills Argument, sondern alle konsequenzialistischen Argumentationsstrategien, unabhängig davon, wie sie im Einzelnen begründet sind (z.B. intuitionistisch), da sie ein objektiv Gutes als Zielzustand setzen und davon ausgehend, die Pflicht, ihn zu realisieren ableiten. ${ }^{52}$

Scheffler hatte behauptet, dass es unklar sei, wie ein praktisches Gesetz einerseits als Forderung der Vernunft, das Gute zu verwirklichen, verstanden werden und gleichzeitig, akteurzentrierte Einschränkungen in Form deontologischer Pflichten verständlich machen kann (Scheffler 1985, 419). Eine methodisch reflektierte Argumentation kann allerdings zeigen, dass Scheffler sich mit dieser Vermutung irrt. Vielmehr sollte durch die Analyse von Mills Beweis deutlich geworden sein, dass es im Gegenteil unklar ist, wie ausgehend von einem Modell maximierender Rationalität und der methodologisch ungeklärten Rede von der Herbeiführung des Guten schließlich überhaupt ein Prinzip des Guten, das nicht nur relativ zur kontingenten

52 Vgl. wiederum die von uns zugrunde gelegte Bestimmung des Konsequenzialismus nach Wiggins (2006, 149f.). 
Präferenzwahl eines Subjekts gültig wäre, ermittelt werden könnte. Da vernünftig Handelnde so gedacht werden müssen, dass sie immer die Frage stellen können, warum das konsequenzialistische Maximierungsprinzip realisiert werden muss, hilft an dieser Stelle Schefflers metaphysischer Verweis auf die angeblich unschuldige Annahme (innocent assumption), dass das Gute dem Bösen immer vorzuziehen und es daher als das Wünschenswertere zu maximieren sei, nicht weiter. Ohne praktische Reflexion auf das Vermögen der freien Präferenzwahl im Verhältnis zu diesem Prinzip bleibt diese Auskunft unvollständig.

Für die methodische Reflexion auf die Erkenntnisbedingungen des Guten war es, wie schon bemerkt, nicht erforderlich, eine von der üblicherweise anerkannten Form der Rationalität abweichende Form praktischer Vernunft ins Spiel zu bringen. Das deontologische Programm, Prinzipien als Forderungen der Beachtung logischer Notwendigkeit in unserem reflektierten Handeln zu begründen, die zugleich das Vermögen der Präferenzwahl einschränken, führt daher auch in keine Paradoxien. Nach unserer methodischen Reflexion gibt es zur Auszeichnung einer möglichen praktischen Erkenntnis als Erkenntnis einer praktischen Notwendigkeit keine andere Alternative außer der reflexiven Prüfung ihrer Erkenntnisbedingungen aus der Perspektive eines praktisch-vernünftig handelnden Subjekts. Hier gilt dann ein binäres Verhältnis im Sinne einer vollständigen Disjunktion: Entweder eine Erkenntnis erfüllt die Erkenntnisbedingung, dann ist sie absolut praktisch notwendig, oder sie erfüllt diese nicht, dann ist diese Erkenntnis nur unter bestimmten Einschränkungen gut, und damit auch die Aufforderung, diese zu realisieren. Die Maximierung des Guten durch eine Handlungsweise, die möglicherweise besser geeignet wäre, dieses zu realisieren, ist gemäß der hier vorgelegten methodologischen Reflexion nicht denkbar. Ein deontologisches Paradox existiert daher nicht. Im Gegenteil müsste ein konsequenzialistisches Konzept begründen, wie ohne subjektive Setzung des Guten qua Entscheidung eines Subjekts überhaupt auch nur irgendeine Handlung als eindeutig gut bzw. praktisch notwendig identifizierbar sein sollte.

Der Konflikt zwischen einer praktischen Vernunft, die als Vermögen verstanden wird, nach Prinzipien zu handeln, und der maximierenden Rationalität, die fordert, immer den besten Weltzustand herbeizuführen, erweist sich somit als nur scheinbarer. Der von Korsgaard (1996, 275) im Zitat, das wir diesem Beitrag vorangestellt haben, festgestellte Denkfehler des Konsequenzialismus, dass es die Aufgabe der Moral sei, etwas in der Welt hervorzubringen, ist einer mangelnden Reflexion auf die Erkenntnisbedingungen des Guten geschuldet. ${ }^{53}$

Funding Open Access funding provided by Projekt DEAL.

Open Access Dieser Artikel wird unter der Creative Commons Namensnennung 4.0 International Lizenz veröffentlicht, welche die Nutzung, Vervielfältigung, Bearbeitung, Verbreitung und Wiedergabe in jeglichem Medium und Format erlaubt, sofern Sie den/die ursprünglichen Autor(en) und die Quelle ordnungsgemäß nennen, einen Link zur Creative Commons Lizenz beifügen und angeben, ob Änderungen vorgenommen wurden.

\footnotetext{
53 Wir danken unseren Kollegen Christoph Hubig, Jan Müller und Sascha Settegast, die frühere Versionen
} dieses Aufsatzes mit uns diskutiert haben. 
Die in diesem Artikel enthaltenen Bilder und sonstiges Drittmaterial unterliegen ebenfalls der genannten Creative Commons Lizenz, sofern sich aus der Abbildungslegende nichts anderes ergibt. Sofern das betreffende Material nicht unter der genannten Creative Commons Lizenz steht und die betreffende Handlung nicht nach gesetzlichen Vorschriften erlaubt ist, ist für die oben aufgeführten Weiterverwendungen des Materials die Einwilligung des jeweiligen Rechteinhabers einzuholen.

Weitere Details zur Lizenz entnehmen Sie bitte der Lizenzinformation auf http://creativecommons.org/ licenses/by/4.0/deed.de.

\section{Anhang}

Wir verstehen unsere von Kant inspirierte Argumentation im Haupttext als eine systematische Reflexion und nicht als Kant-Interpretation. Ihr Geltungsanspruch muss daher unabhängig von unseren exegetischen Einlassungen geprüft werden. Dennoch wollen wir abschließend für eine speziell an Kant interessierte Leserschaft Kants methodologische Einlassung zum „Paradoxon“ in den Gesamtzusammenhang seiner Argumentation in GMS und $\mathrm{KpV}$ einordnen. Zur Erläuterung der Argumentation der KpV-Analytik folgen wir Michael Wolff (2009):

1. Ausgangspunkt ist die Begriffsbestimmung von Gesetz (KpV, AA V, §1 u. GMS Vorrede)

2. [Erläuterung der Problemstellung:] Die Deklaration (KpV, AA V, §1) bzw. Definition von „Gesetz“ (GMS, AA IV, 389) erfolgt als Antwort auf das Problem, die Denkbarkeit der Notwendigkeit einer Handlung nicht relativ zur Präferenzwahl eines Zwecks, sondern aus Achtung nur gegen die Forderung der Notwendigkeit einer Handlung zu ermitteln.

3. [Überblick zum gesamten Argumentationsverfahren:] Analytische Urteile führen auf die Formel des einzigen Grundgesetzes der reinen praktischen Vernunft (KpV: deklarierter formaler Begriff von Gesetz, Analyse und indirekte Argumente, inhaltlicher Begriff des Grundgesetzes, Postulat) (Wolff 2009). In GMS erfolgt die analytische Ableitung der Formel eines kategorischen Imperativs aus seinem Begriff (GMS, AA IV, 420)..$^{4}$

4. [Begriffsbestimmung:] ,gut“ ist Prädikat der Erkenntnis des praktisch Notwendigen (GMS, AA IV, 412).

5. [semantische Umformung:] Als „gut“ ist nur das erkennbar, was mindestens der Formel des Grundgesetzes oder des kategorischen Imperativs entspricht. Alles andere ist nur relativ dazu als ,gut" erkennbar.

6. [methodologische Erläuterung I:] Die Formel wird analytisch entwickelt, sie ist jedoch selbst ein synthetisch-praktischer Satz a priori (bei Mill nötigt die Formel des Nützlichkeitsprinzips dagegen analytisch durch zu vermeidenden Widerspruch).

7. [methodologische Erläuterung II:] Ohne Beachtung der Formel ist keine Prädikation mit ,gut" möglich bzw. keine Erkenntnis des Guten denkbar (s. Definition bei 4) - es handelt sich bei der Erkenntnis gemäß der Formel in KpV und GMS um ei-

\footnotetext{
${ }^{54}$ Die Berechtigung des Begriffsgebrauchs wird durch transzendentale Deduktion in GMS, AA IV, III und durch Postulat der Formel als mögliche Forderung in KpV, AA V, §7 (Anmerkung) nachgewiesen.
} 
ne Affirmation im logischen Raum, über deren Notwendigkeit in jeder Handlung, die mit dem Erkenntnisprädikat ,gut“ operiert, Vergewisserung möglich ist. Die Bedingungen eines praktischen Gesetzes sind zu beachten, wenn eine Prädikation mit ,gut" durchführbar sein soll.

8. [Pointe des „Paradoxon der Methode“:] Der Versuch der argumentativen Widerlegung der Begründung bzw. Gültigkeit der Formel lässt sich durch ein Retorsionsargument zurückweisen. Dieses führt beim Opponenten auf das Erfordernis, einen Alternativbegriff von ,gut“ einzuführen, der die Bedingung einer Erkenntnis des praktisch Notwendigen ebenso oder stärker erfüllt. Dies ist zumindest im utilitaristischen Konsequenzialismus von Mill (und Scheffler), wie wir gesehen haben, strukturell nicht möglich: Die Erkenntnis des praktisch Notwendigen bleibt dort hinsichtlich der Erkenntnis durch den Akteur notwendig kontingent.

\section{Literatur}

Aristoteles. 2006. Nikomachische Ethik. Übersetzt und herausgegeben von Ursula Wolf. Reinbek bei Hamburg: Rowohlt.

Bambauer, Christoph. 2019. Die praktische Notwendigkeit des Guten. Handlungstheoretische Ethikbegründung im Ausgang von Gewirth und Korsgaard. Paderborn: Mentis.

Brink, David O. 1992. Mill's Deliberative Utilitarianism. Philosophy \& Public Affairs 21.1: 67-103.

Crisp, Roger. 2017. The Cosmos of Duty. Henry Sidgwick's Methods of Ethics. Oxford: University Press.

Engstrom, Stephen. 1997. Kant's Conception of Practical Wisdom. In Kant-Studien 88: 16-43.

Engstrom, Stephen. 2009. The Form of Practical Knowledge. A Study of the Categorial Imperative. Cambridge/London: Harvard University Press.

Frankena, William K.: Der naturalistische Fehlschluss. In Metaethik. Klassische Texte, hrsg. v. Bert Heinrichs und Jan-Hendrik Heinrichs, 48-65. Berlin: Suhrkamp.

Fyfe, A.T. 2012. John Stuart Mills Beweis des Utilitarismus. In Die 100 wichtigsten philosophischen Argumente, hrsg. v. Michael Bruce und Steven Barbone, 224-229. Darmstadt: Wissenschaftliche Buchgesellschaft.

Gewirth, Alan. 1981. Reason and Morality. Chicago: University Press.

Grundmann, Thomas. 1994. Analytische Transzendentalphilosophie. Eine Kritik. Paderborn: Schöningh.

Hegel, Georg W. F. 1820/1986. Grundlinien der Philosophie des Rechts. Frankfurt a. M.: Suhrkamp.

Heuer, Ulrike. 2011. The paradox of deontology, revisited. In Oxford Studies in Normative Ethics, ed. Mark Timmons, 236-267. Oxford: University Press.

Heydt, Colin. 2006. Rethinking Mill's Ethics. Character and Aesthetic Education. London: Continuum Press.

Hume, David. 1751/2003. Untersuchung über die Prinzipien der Moral. Hamburg: Meiner.

Kamm, Frances M. 2007. Intricate Ethics. Rights, Responsibilities, and Permissible Harm. Oxford: University Press.

Kant, Immanuel. 1785/1903. Grundlegung zur Metaphysik der Sitten. In Kants gesammelte Schriften. Hrsg. von der Königlich Preußischen Akademie der Wissenschaften. Bd. IV. Hrsg. von Paul Menzer. Berlin 1903.

Kant, Immanuel. 1788/1961. Kritik der praktischen Vernunft. In Kants gesammelte Schriften. Hrsg. von der Königlich Preußischen Akademie der Wissenschaften. Bd. V. Hrsg. von Paul Natorp. Berlin 1961, Nachdruck 1968.

Kobusch, Theo. 2016. Die praktischen Elementarbegriffe als Modi der Willensbestimmung. In „Die Kategorie der Freiheit“ in Kants praktischer Philosophie, hrsg. v. Stephan Zimmermann, 17-75. Berlin/ Boston: de Gruyter.

Korsgaard, Christine M. 1996. The Reasons We Can Share. In Creating the Kingdom of Ends, 275-310. Cambridge: University Press.

Mill, John Stuart. 2006. Utilitarianism. Der Utilitarismus. Englisch/Deutsch. Übersetzt und herausgegeben von Dieter Birnbacher. Stuttgart: Reclam.

Moore, George E. 1970. Principia Ethica. Stuttgart: Reclam.

Moore, George E. 1975. Grundprobleme der Ethik. München: Beck. 
Müller, Olaf L. 2003. John Stuart Mills Argument für den Utilitarismus. Ein plausibler Weg zwischen Metaphysik und Nihilismus? In Geschichte der Ethik: Jahrbuch für Philosophiegeschichte und logische Analyse 6, hrsg. v. Uwe Meixner und Albert Newen, 167-191. Paderborn: Mentis.

Nozick, Robert. 2013. Anarchy, State, Utopia. New York: Basic Books.

Rawls, John. 1988. Theorie der Gerechtigkeit. Frankfurt a. M.: Suhrkamp.

Richter, Philipp. 2020. Von den Aporien der praktischen Vernunft zur Erkenntnis der praktischen Notwendigkeit. In Allgemeine Zeitschrift für Philosophie 3/2020, Schwerpunkt: Praktisches Wissen. [im Erscheinen]

Sayre-McCord, Geoffrey. 2001. Mill's Proof of the Principle of Utility: A More than Half-Hearted Defense. Philosophy \& Policy 18/2: 330-360.

Scanlon, Thomas M. 2000. What we Owe to Each Other. Cambridge/MA: Harvard University Press.

Scheffler, Samuel. 1985. Agent-centred Restrictions, Rationality, and the Virtues. Mind 94 375: 409-419.

Scheffler, Samuel. 1988. Introduction. In Consequentialism and its Critics, ed. Samuel Scheffler, 1-13. Oxford: Oxford University Press.

Scheffler, Samuel. 1991. The Rejection of Consequentialism. A Philosophical Investigation of the Considerations underlying Rival Moral Conceptions. Oxford: Clarendon Press.

Sensen, Oliver. 2013. „Kant's Constructivism“. In Constructivism in Ethics, ed. Carla Bagnoli, 63-81. Cambridge: Cambridge University Press.

Sidgwick, Henry. 1981. The Methods of Ethics. Indianapolis/IN: Hackett Publishing Company.

Skorupski, John. 1991. John Stuart Mill. London: Routledge.

Steigleder, Klaus. 1997. Gewirth und die Begründung der normativen Ethik. Zeitschrift für philosophische Forschung 51: 250-266.

Steigleder, Klaus. 2002. Kants Moralphilosophie. Die Selbstbezüglichkeit reiner praktischer Vernunft. Stuttgart/Weimar: Metzler.

Wiggins, David. 2006. Ethics. Twelve Lectures on the Philosophy of Morality. London: Penguin Books.

Wolf, Jean-Claude. 2012. John Stuart Mills „Utilitarismus“. Ein kritischer Kommentar. Freiburg im Breisgau/München: Alber.

Wolff, Michael. 2009. Warum das Faktum der Vernunft ein Faktum ist. Auflösung einiger Verständnisschwierigkeiten in Kants Grundlegung der Moral. Deutsche Zeitschrift für Philosophie 57.4: 511549 . 